MATHEMATICS OF COMPUTATION

Volume 80, Number 273, January 2011, Pages 11-41

S $0025-5718(2010) 02364-6$

Article electronically published on July 26, 2010

\title{
A TWO-LEVEL ENRICHED FINITE ELEMENT METHOD FOR A MIXED PROBLEM
}

\author{
ALEJANDRO ALLENDES, GABRIEL R. BARRENECHEA, ERWIN HERNÁNDEZ, \\ AND FRÉDÉRIC VALENTIN
}

\begin{abstract}
The simplest pair of spaces $\mathbb{P}_{1} / \mathbb{P}_{0}$ is made inf-sup stable for the mixed form of the Darcy equation. The key ingredient is to enhance the finite element spaces inside a Petrov-Galerkin framework with functions satisfying element-wise local Darcy problems with right hand sides depending on the residuals over elements and edges. The enriched method is symmetric, locally mass conservative and keeps the degrees of freedom of the original interpolation spaces. First, we assume local enrichments exactly computed and we prove uniqueness and optimal error estimates in natural norms. Then, a low cost two-level finite element method is proposed to effectively obtain enhancing basis functions. The approach lays on a two-scale numerical analysis and shows that well-posedness and optimality is kept, despite the second level numerical approximation. Several numerical experiments validate the theoretical results and compares (favourably in some cases) our results with the classical RaviartThomas element.
\end{abstract}

\section{INTRODUCTION}

The selection of finite dimensional spaces for the Galerkin method demands careful attention when it comes to solving the weak form of mixed boundary value problems. In fact, mixed problems can be handled by polynomial interpolations if the pair of spaces fulfill the well-known inf-sup condition [11. This leads to some very popular choices, such as equal order interpolation spaces and the simplest element $\mathbb{P}_{1} / \mathbb{P}_{0}$ to be out of reach, or it prevents nodal values to be chosen as degrees of freedom if some physical properties (such as local conservation of mass) are to be satisfied by the numerical method (cf. 9]).

On the quest to systematically build stable and accurate finite element methods, numerical solutions have been formally decomposed into solved and unsolved parts with respect to a fixed mesh. Roughly, the resolved part solves the original weak form on a given finite element space regardless of any numerical drawback, while

Received by the editor October 10, 2008 and, in revised form, July 1, 2009.

2010 Mathematics Subject Classification. Primary 65N30, 65N12; Secondary 76S99.

Key words and phrases. Darcy flow, enriched finite element method, Petrov-Galerkin approach, mass conservation, two-level finite element method.

The second author was partially supported by Starter's Grant, Faculty of Sciences, University of Strathclyde.

The third author was supported by CONICYT Chile, through FONDECYT Project No. 1070276 and by Universidad Santa María through project No. DGIP-USM 120851.

The fourth author was supported by CNPq /Brazil Grant No. 304051/2006-3, FAPERJ/Brazil Grant No. E-26/100.519/2007.

(C)2010 American Mathematical Society Reverts to public domain 28 years from publication 
the unsolved part comes into play to make the complete solution free of numerical trouble and recover missed physical properties as well. The final methods are said to incorporate "missing scales" not captured by the mesh, and so, are named multi-scale methods. Different approaches to model the unsolved scales have been proposed over the past years, among which we might mention the Variational MultiScale method (VMS) [24] and variations of it [3, 16, the Residual-Free-Bubble approach (RFB) [14, 27, 12 and its practical implementation using a two-level method [22, the Heterogeneous Multi-Scale Methods (HMM) [18, and recently, Petrov-Galerkin Enriched Methods (PGEM) (see [21, 20, 5, and 11 for a survey). Overall, algorithms are closely related by making the unsolved contribution dependent on geometrical aspects of the mesh, the interpolation space choice and the boundary value problem itself. In parallel, RFB and PGEM have been systematically related to stabilized methods and stabilization parameters been obtained with respect to the mean value of enriching basis functions [4, 13, 7, 8, 2].

This work addresses the subject for the Darcy equation, a model that appears in porous media, in the Petrov-Galerkin enriched framework. The PGEM has been introduced as a way to incorporate the edge residual contribution into the unsolved scale modeling, an aspect neglected by the RFB and responsible for the non-physical oscillations in the numerical solutions. In 5 the standard trial and test finite element spaces $\mathbb{P}_{1} / \mathbb{P}_{0}$ are differently enriched. The latter remains enhanced with bubble functions as in the RFB method, but now the trial space incorporates functions driven by the Darcy operator, depending on the residual of the equation in each element and on each edge of the triangulation. The boundary conditions for the local problems are set in such a way as to ensure the continuity of both the normal component of the enriched velocity (strongly) and pressure (weakly). Thereby, the desired features of the $\mathbb{P}_{1} / \mathbb{P}_{0}$ element are preserved, namely its simplicity along with its nodal-valued degrees of freedom for the velocity and the locally mass conservative velocity field.

Two non-symmetric enriched methods were proposed in [5] and extensive numerical validations have been carried out for one of the methods in which the solution to the local problems is known analytically. However, neither numerical analysis nor numerical validations have been proposed for the original (two level) method from [5. This work aims at overcoming these shortcomings and introducing, moreover, a symmetric method. Keeping the simplest element $\mathbb{P}_{1} / \mathbb{P}_{0}$ as the target spaces, and assuming that enriching local problems are exactly solved, we prove existence and uniqueness for both the original non-symmetric and the symmetric methods, as well as optimal error estimates in natural norms. In fact, we show that the leading error between both methods tends faster to zero than the error itself when the characteristic length of the mesh goes to zero.

Next, the semidiscrete method analyzed before is completely discretized using a two-level approach incorporating numerically computed unsolved scales into the enriched method. The order of convergence is not affected by this approximation under mild conditions on the fine scale discretization. It is worth mentioning that, up to our knowledge, few works incorporate fine scales approximation in numerical analysis. Moreover, a low-cost procedure has been proposed to effectively incorporate the subscales. As a matter of fact, as fine scale mesh, one single $\mathbb{P}_{1}$ element is used throughout all the numerical experiments, keeping optimal convergence. 
Although not theoretically proved, numerical results highlight quadratic convergence for the velocity in the $L^{2}$ norm, a feature that is clearly not expected for the Raviart-Thomas element [26, 11]. The comparison with the standard RaviartThomas method is pushed further and allows us to outline the main features of the two-level enriched method; namely:

- has a lower number of degrees of freedom for a fixed mesh;

- induces a symmetric linear system (if we were disposed to relax the symmetry requirement, then an equivalent non-symmetric but positive definite system may be proposed);

- keeps nodal-valued degrees of freedom for the velocity;

- is locally mass conservative.

The remainder of the paper is as follows: the current section ends with notation and preliminary results; for the sake of completeness the derivation of the PGEM in [5] is revisited in Section 2] and then, the resulting methods are mathematically analyzed in Section 3. Section 4 is devoted to the two-level enriched method which is numerically validated in Section 5 . Conclusions are drawn in Section 6 .

1.1. Notation. This section introduces definitions and notation used throughout. In what follows, $\Omega$ denotes an open bounded domain in $\mathbb{R}^{2}$ with polygonal boundary $\partial \Omega$, and $\mathbf{x}=\left(x_{1}, x_{2}\right)$ is a typical point in $\Omega$. As usual, $L^{2}(\Omega)$ is the space of square integrable functions over $\Omega, L_{0}^{2}(\Omega)$ represents functions belonging to $L^{2}(\Omega)$ with zero average in $\Omega$, and $H(\operatorname{div}, \Omega)(H(\mathrm{curl}, \Omega))$ is composed by functions that belong to $L^{2}(\Omega)^{2}$ with divergence $($ curl $)$ in $L^{2}(\Omega)$. The space $H_{0}(d i v, \Omega)$ stands for the space of functions belonging to $H(\operatorname{div}, \Omega)$ with vanishing normal component on $\partial \Omega$. Finally, $(\cdot, \cdot)_{D}$ stands for the inner product in $L^{2}(D)$ (or in $L^{2}(D)^{2}$, when necessary), and $\|\cdot\|_{s, D}\left(|\cdot|_{s, D}\right)$ the norm (seminorm) in $H^{s}(D)$ (or $\left.H^{s}(D)^{2}\right),|\cdot|_{0, D}=$ $\|\cdot\|_{0, D}$, and $\|\cdot\|_{d i v, D}$ the norm in $H(\operatorname{div}, D)$.

From now on we denote by $\left\{\mathcal{T}_{H}\right\}$ a family of regular triangulations of $\bar{\Omega}$ built using triangles $K$ with boundary $\partial K$ composed by edges $F$. The set of internal edges of the triangulation $\mathcal{T}_{H}$ is denoted by $\mathcal{E}_{H}$. The characteristic length of $K$ and $F$ are denoted by $H_{K}$ and $H_{F}$, respectively, and $H:=\max \left\{H_{K}: K \in \mathcal{T}_{H}\right\}$, and due to the mesh regularity there exists a positive constant $C$ such that $H_{F} \leq$ $H_{K} \leq C H_{F}$, for all $F \subseteq \partial K$. Also, for each $F=K \cap K^{\prime} \in \mathcal{E}_{H}$ we choose, once and for all, a unit normal vector $\boldsymbol{n}$ which coincides with the unit outward normal vector when $F \subseteq \partial \Omega$. The standard outward normal vector at the edge $F$ with respect to the element $K$ is denoted by $\boldsymbol{n}_{F}^{K}$. Moreover, for a function $q$, $q \rrbracket$ denotes its jump, defined by (see Figure 1)

$$
\llbracket q \rrbracket(\mathbf{x}):=\lim _{\delta \rightarrow 0^{+}} q(\mathbf{x}+\delta \boldsymbol{n})-\lim _{\delta \rightarrow 0^{-}} q(\mathbf{x}+\delta \boldsymbol{n}),
$$

and $\llbracket q \rrbracket=0$ if $F \subseteq \partial \Omega$. We finally introduce the following broken spaces:

$$
\begin{aligned}
H_{0}\left(\operatorname{div}, \mathcal{T}_{H}\right) & :=\left\{\boldsymbol{v} \in L^{2}(\Omega)^{2}:\left.\boldsymbol{v}\right|_{K} \in H_{0}(\operatorname{div}, K) \forall K \in \mathcal{T}_{H}\right\}, \\
L_{0}^{2}\left(\mathcal{T}_{H}\right) & :=\left\{q \in L^{2}(\Omega):\left.q\right|_{K} \in L_{0}^{2}(K) \forall K \in \mathcal{T}_{H}\right\} .
\end{aligned}
$$

1.2. Preliminaries. In this work we consider the following Darcy problem: Find $(\boldsymbol{u}, p)$ such that

$$
\begin{gathered}
\sigma \boldsymbol{u}+\nabla p=\boldsymbol{f}, \quad \nabla \cdot \boldsymbol{u}=g \quad \text { in } \Omega \\
\boldsymbol{u} \cdot \boldsymbol{n}=0 \quad \text { on } \partial \Omega,
\end{gathered}
$$




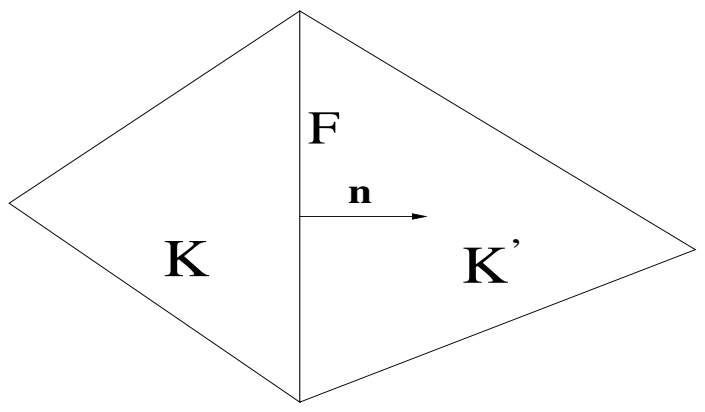

FiguRE 1. The normal vector.

where $\sigma=\frac{\mu}{\kappa} \in \mathbb{R}^{+}$is assumed constant in $\Omega$, with $\mu$ and $\kappa$ denoting the viscosity and permeability, respectively. Here, $\boldsymbol{u}$ is the so-called Darcy velocity, $p$ is the pressure, and $\boldsymbol{f}$ and $g$ are given source terms. We suppose $\boldsymbol{f}$ is piecewise constant since it is usually related to the gravity force (see the numerical experiments for an extension to the general case). Moreover, we assume that the usual compatibility condition

$$
\int_{\Omega} g=0
$$

holds.

The standard mixed variational formulation associated with (1.4) reads: Find $(\boldsymbol{u}, p) \in H_{0}(\operatorname{div}, \Omega) \times L_{0}^{2}(\Omega)$ such that

$$
\mathbf{A}((\boldsymbol{u}, p),(\boldsymbol{v}, q))=\boldsymbol{L}(\boldsymbol{v}, q) \quad \forall(\boldsymbol{v}, q) \in H_{0}(\operatorname{div}, \Omega) \times L_{0}^{2}(\Omega),
$$

where

$\mathbf{A}((\boldsymbol{u}, p),(\boldsymbol{v}, q)):=(\sigma \boldsymbol{u}, \boldsymbol{v})_{\Omega}-(p, \nabla \cdot \boldsymbol{v})_{\Omega}-(q, \nabla \cdot \boldsymbol{u})_{\Omega}, \quad \boldsymbol{L}(\boldsymbol{v}, q):=(\boldsymbol{f}, \boldsymbol{v})_{\Omega}-(g, q)_{\Omega}$.

The well-posedness of (1.5) follows from the classical Babuska-Brezzi theory for variational problems with constraints (see [11] for details).

\section{The ENRICHED Finite ElEMENT METHOD}

We start generalizing the derivation carried out in [5]. We introduce the standard finite element space $\mathbf{V}_{H}:=\left[V_{H}\right]^{2} \cap H_{0}(\operatorname{div}, \Omega)$ for the velocity variable, where

$$
V_{H}:=\left\{v \in C^{0}(\bar{\Omega}):\left.v\right|_{K} \in \mathbb{P}_{1}(K), \forall K \in \mathcal{T}_{H}\right\},
$$

whereas the pressure is discretized using the space

$$
Q_{H}:=\left\{q_{0} \in L_{0}^{2}(\Omega):\left.q_{0}\right|_{K} \in \mathbb{P}_{0}(K) \forall K \in \mathcal{T}_{H}\right\} .
$$

Our starting point is the following Petrov-Galerkin scheme: Find $\boldsymbol{u}_{H}=\boldsymbol{u}_{1}+\boldsymbol{u}_{e} \in$ $\mathbf{V}_{H}+H_{0}(\operatorname{div}, \Omega)$ and $p_{H}=p_{0}+p_{e} \in Q_{H} \oplus L_{0}^{2}\left(\mathcal{T}_{H}\right)$ such that

$$
\mathbf{A}\left(\left(\boldsymbol{u}_{H}, p_{H}\right),\left(\boldsymbol{v}_{H}, q_{H}\right)\right)=\boldsymbol{L}\left(\boldsymbol{v}_{H}, q_{H}\right),
$$

for all $\boldsymbol{v}_{H}=\boldsymbol{v}_{1}+\boldsymbol{v}_{b} \in \mathbf{V}_{H} \oplus H_{0}\left(d i v, \mathcal{T}_{H}\right)$ and for all $q_{H}=q_{0}+q_{b} \in Q_{H} \oplus L_{0}^{2}\left(\mathcal{T}_{H}\right)$. 
Thanks to the choice made for the test function enrichment space, we can split (2.3) into the following system:

$$
\mathbf{A}\left(\left(\boldsymbol{u}_{1}+\boldsymbol{u}_{e}, p_{0}+p_{e}\right),\left(\boldsymbol{v}_{1}, q_{0}\right)\right)=\boldsymbol{L}\left(\boldsymbol{v}_{1}, q_{0}\right) \quad \forall\left(\boldsymbol{v}_{1}, q_{0}\right) \in \mathbf{V}_{H} \times Q_{H},
$$

$$
\mathbf{A}\left(\left(\boldsymbol{u}_{1}+\boldsymbol{u}_{e}, p_{0}+p_{e}\right),\left(\boldsymbol{v}_{b}, q_{b}\right)\right)=\boldsymbol{L}\left(\boldsymbol{v}_{b}, q_{b}\right) \quad \forall\left(\boldsymbol{v}_{b}, q_{b}\right) \in H_{0}\left(\operatorname{div}, \mathcal{T}_{H}\right) \times L_{0}^{2}\left(\mathcal{T}_{H}\right) .
$$

First, considering test functions $\left(\boldsymbol{v}_{b}, q_{b}\right)$ supported in a single element, we see that (2.5) is the weak form of the following strong problem for $\left(\boldsymbol{u}_{e}, p_{e}\right)$ :

$$
\sigma \boldsymbol{u}_{e}+\nabla p_{e}=\boldsymbol{f}-\sigma \boldsymbol{u}_{1}, \quad \nabla \cdot \boldsymbol{u}_{e}=g+C_{K} \quad \text { in } K,
$$

where $C_{K} \in \mathbb{R}$ is, a priori, free. Now, to close this problem we impose the following boundary condition on $\boldsymbol{u}_{e}$ (see [5]):

$$
\sigma \boldsymbol{u}_{e} \cdot \boldsymbol{n}=\alpha_{F} \int_{F} \llbracket p_{0} \rrbracket
$$

on each $F \subseteq \partial K \cap \Omega$, and $\boldsymbol{u}_{e} \cdot \boldsymbol{n}=0$ on $F \subseteq \partial \Omega$. Here, $\alpha_{F}$ stands for a constant not depending on $H$ or $\sigma$, but that can vary on each $F \in \mathcal{E}_{H}$. This choice for boundary condition leads us to fix the constant $C_{K}$, which is given by

$$
C_{K}=\overline{\llbracket p_{0} \rrbracket}-\Pi_{K}(g):=\frac{1}{|K|} \sum_{i=1}^{3} \frac{\alpha_{F_{i}} H_{F_{i}}}{\sigma} \int_{F_{i}} \llbracket p_{0} \rrbracket \boldsymbol{n} \cdot \boldsymbol{n}_{F_{i}}^{K}-\frac{\int_{K} g}{|K|} .
$$

Remark 2.1. An alternative method was also derived in [5] by proposing a different boundary condition (see also [6] for the convergence analysis of a related approach).

and

$$
\begin{gathered}
\sigma \boldsymbol{u}_{e}^{g}+\nabla p_{e}^{g}=\mathbf{0}, \quad \nabla \cdot \boldsymbol{u}_{e}^{g}=g-\Pi_{K}(g) \quad \text { in } K, \\
\sigma \boldsymbol{u}_{e}^{g} \cdot \boldsymbol{n}=0 \quad \text { on } \quad \text { each } F \subseteq \partial K,
\end{gathered}
$$

respectively, here, to ensure uniqueness, we seek for $p_{e}^{M}, p_{e}^{D}$ and $p_{e}^{g}$ in $L_{0}^{2}(K)$. Also, we define the solution operators associated to the above problems such that we can write

$$
\begin{gathered}
\left(\boldsymbol{u}_{e}^{M}, p_{e}^{M}\right)=\left(\mathcal{M}_{K}^{u}\left(\boldsymbol{f}-\sigma \boldsymbol{u}_{1}\right), \mathcal{M}_{K}^{p}\left(\boldsymbol{f}-\sigma \boldsymbol{u}_{1}\right)\right), \\
\left(\boldsymbol{u}_{e}^{D}, p_{e}^{D}\right)=\left(\mathcal{D}_{K}^{u}\left(\llbracket p_{0} \rrbracket\right), \mathcal{D}_{K}^{p}\left(\llbracket p_{0} \rrbracket\right)\right), \\
\left(\boldsymbol{u}_{e}^{g}, p_{e}^{g}\right)=\left(\mathcal{G}_{K}^{u}\left(g-\Pi_{K}(g)\right), \mathcal{G}_{K}^{p}\left(g-\Pi_{K}(g)\right)\right),
\end{gathered}
$$

and we remark that the local problem (2.9) may be solved analytically (cf. [5]), which leads to the following explicit expression for $\boldsymbol{u}_{e}^{D}$ :

$$
\boldsymbol{u}_{e}^{D}=\mathcal{D}_{K}^{u}\left(\llbracket p_{0} \rrbracket\right)=\sum_{F \subseteq \partial K} \frac{\alpha_{F} H_{F}}{\sigma} \llbracket p_{0} \rrbracket_{F} \boldsymbol{\varphi}_{F},
$$


where $\varphi_{F}$ is the Raviart-Thomas' basis function defined by

$$
\varphi_{F}(\mathbf{x})= \pm \frac{H_{F}}{2|K|}\left(\mathbf{x}-\mathbf{x}_{F}\right)
$$

$\mathbf{x}_{F}$ denotes the node opposite to the edge $F$ and in which the sign on the RaviartThomas basis function $\varphi_{F}$ depends on whether the normal vector $\boldsymbol{n}$ on $F \subseteq \partial K$ points inwards or outwards of $K$.

Now, after the full characterization of $\left(\boldsymbol{u}_{e}, p_{e}\right)$ given by (2.11)-(2.13), we come back to (2.4). First, since $p_{e} \in L_{0}^{2}(K)$ and $\left.\nabla \cdot \boldsymbol{v}_{1}\right|_{K} \in \mathbb{R}$ we obtain

$$
\left(p_{e}, \nabla \cdot \boldsymbol{v}_{1}\right)_{K}=0 \text { for all } K \in \mathcal{T}_{H} \text {. }
$$

Therefore, problem (2.4) becomes: Find $\left(\boldsymbol{u}_{1}, p_{0}\right) \in \mathbf{V}_{H} \times Q_{H}$ such that

$$
\mathbf{A}\left(\left(\boldsymbol{u}_{1}+\boldsymbol{u}_{e}, p_{0}\right),\left(\boldsymbol{v}_{1}, q_{0}\right)\right)=\boldsymbol{L}\left(\boldsymbol{v}_{1}, q_{0}\right) \quad \forall\left(\boldsymbol{v}_{1}, q_{0}\right) \in \mathbf{V}_{H} \times Q_{H},
$$

or, equivalently using the fact that $\nabla \cdot \boldsymbol{u}_{e}^{M}=0$ and that $\nabla \cdot \boldsymbol{u}_{e}^{g}=g-\Pi_{K}(g)$ is orthogonal to any constant in $K$,

$$
\begin{gathered}
\sigma\left(\boldsymbol{u}_{1}+\boldsymbol{u}_{e}^{M}+\boldsymbol{u}_{e}^{D}+\boldsymbol{u}_{e}^{g}, \boldsymbol{v}_{1}\right)_{\Omega}-\left(p_{0}, \nabla \cdot \boldsymbol{v}_{1}\right)_{\Omega}-\left(q_{0}, \nabla \cdot \boldsymbol{u}_{1}\right)_{\Omega} \\
-\left(q_{0}, \nabla \cdot \boldsymbol{u}_{e}^{D}\right)_{\Omega}=\boldsymbol{L}\left(\boldsymbol{v}_{1}, q_{0}\right),
\end{gathered}
$$

for all $\left(\boldsymbol{v}_{1}, q_{0}\right) \in \mathbf{V}_{H} \times Q_{H}$. Next, integrating by parts in each $K \in \mathcal{T}_{H}$ and using the boundary condition (2.7) we obtain

$$
\sum_{K \in \mathcal{T}_{H}}\left(q_{0}, \nabla \cdot \boldsymbol{u}_{e}^{D}\right)_{K}=\sum_{F \in \mathcal{E}_{H}} \tau_{F}\left(\llbracket p_{0} \rrbracket, \llbracket q_{0} \rrbracket\right)_{F},
$$

where

$$
\tau_{F}:=\frac{\alpha_{F} H_{F}}{\sigma} .
$$

Remark 2.2. The term related to $\boldsymbol{f}$ in (2.11) vanishes. Indeed, since $\boldsymbol{f}$ is constant in $K$, it is easy to realize that $\mathcal{M}_{K}^{u} \boldsymbol{f}=0$, which leads to $\boldsymbol{u}_{e}^{M}=-\sigma \mathcal{M}_{K}^{u}\left(\boldsymbol{u}_{1}\right)$.

Finally, based on the previous remark, and replacing (2.19) and (2.11)-(2.13) in (2.17), we arrive at the following form of our enriched method: Find $\left(\boldsymbol{u}_{1}, p_{0}\right) \in$ $\mathbf{V}_{H} \times Q_{H}$ such that

$$
\begin{gathered}
\mathbf{A}\left(\left(\boldsymbol{u}_{1}-\sigma \mathcal{M}_{K}^{u}\left(\boldsymbol{u}_{1}\right), p_{0}\right),\left(\boldsymbol{v}_{1}, q_{0}\right)\right)+\sum_{K \in \mathcal{T}_{H}}\left(\mathcal{D}_{K}^{u}\left(\llbracket p_{0} \rrbracket\right), \sigma \boldsymbol{v}_{1}\right)_{K} \\
-\sum_{F \in \mathcal{E}_{H}} \tau_{F}\left(\llbracket p_{0} \rrbracket, \llbracket q_{0} \rrbracket\right)_{F}=\boldsymbol{L}\left(\boldsymbol{v}_{1}, q_{0}\right)-\sum_{K \in \mathcal{T}_{H}}\left(\mathcal{G}_{K}^{u}\left(g-\Pi_{K}(g)\right), \sigma \boldsymbol{v}_{1}\right)_{K},
\end{gathered}
$$

for all $\left(\boldsymbol{v}_{1}, q_{0}\right) \in \mathbf{V}_{H} \times Q_{H}$. Since our aim is to derive a symmetric method, in the following lemma we further explore the properties of the operator $\mathcal{M}_{K}^{u}$.

Lemma 2.3. The linear operator $\sigma \mathcal{M}_{K}^{u}$ is an orthogonal projection with respect to the $L^{2}(K)^{2}$ inner product. More precisely, for all $\boldsymbol{v} \in L^{2}(K)^{2}$

$$
\left(\boldsymbol{v}-\sigma \mathcal{M}_{K}^{u}(\boldsymbol{v}), \boldsymbol{w}\right)_{K}=0,
$$

for all $\boldsymbol{w} \in H_{0}($ div,$K)$ such that $\nabla \cdot \boldsymbol{w}=0$ in $K$. Moreover,

$$
\left(\mathcal{M}_{K}^{u}(\boldsymbol{v}), \nabla \psi\right)_{K}=0 \quad \forall \psi \in H^{1}(K) .
$$


Proof. To prove (2.21) we multiply (2.8) by a function $\boldsymbol{w} \in H_{0}(\operatorname{div}, K)$ such that $\nabla \cdot \boldsymbol{w}=0$ in $K$ and we integrate by parts to obtain

$$
\left(\sigma \mathcal{M}_{K}^{u}(\boldsymbol{v}), \boldsymbol{w}\right)_{K}-\left(p_{e}^{M}, \nabla \cdot \boldsymbol{w}\right)_{K}+\left(p_{e}^{M}, \boldsymbol{w} \cdot \boldsymbol{n}\right)_{\partial K}=(\boldsymbol{v}, \boldsymbol{w})_{K},
$$

and the result follows by applying the properties of $\boldsymbol{w}$. Finally, integrating by parts and using the fact that $\mathcal{M}_{K}^{u}(\boldsymbol{v})$ has a vanishing divergence and normal component (2.22) follows.

Remark 2.4. Since $p_{e}^{M} \in L_{0}^{2}(K)$, then (2.21) is also valid if $\nabla \cdot \boldsymbol{w} \in \mathbb{R}$ in $K$.

Using the previous lemma and the fact that all Raviart-Thomas' functions are gradients, we can give the following presentation for our method: Find $\left(\boldsymbol{u}_{1}, p_{0}\right) \in$ $\mathbf{V}_{H} \times Q_{H}$ such that

$$
\boldsymbol{B}_{n s}\left(\left(\boldsymbol{u}_{1}, p_{0}\right),\left(\boldsymbol{v}_{1}, q_{0}\right)\right)=\boldsymbol{L}\left(\boldsymbol{v}_{1}, q_{0}\right)-\sum_{K \in \mathcal{T}_{H}}\left(\mathcal{G}_{K}^{u}\left(g-\Pi_{K}(g)\right), \sigma \boldsymbol{v}_{1}\right)_{K},
$$

for all $\left(\boldsymbol{v}_{1}, q_{0}\right) \in \mathbf{V}_{H} \times Q_{H}$, where

$$
\begin{aligned}
\boldsymbol{B}_{n s}\left(\left(\boldsymbol{u}_{1}, p_{0}\right),\left(\boldsymbol{v}_{1}, q_{0}\right)\right):= & \mathbf{A}\left(\left(\rho\left(\boldsymbol{u}_{1}\right), p_{0}\right),\left(\rho\left(\boldsymbol{v}_{1}\right), q_{0}\right)\right) \\
& +\sum_{K \in \mathcal{T}_{H}}\left(\mathcal{D}_{K}^{u}\left(\llbracket p_{0} \rrbracket\right), \sigma \rho\left(\boldsymbol{v}_{1}\right)\right)_{K}-\sum_{F \in \mathcal{E}_{H}} \tau_{F}\left(\llbracket p_{0} \rrbracket, \llbracket q_{0} \rrbracket\right)_{F} .
\end{aligned}
$$

To simplify the notation we have noted

$$
\left.\rho(\boldsymbol{v})\right|_{K}:=\left(\boldsymbol{I}-\sigma \mathcal{M}_{K}^{u}\right)(\boldsymbol{v}),
$$

where $\boldsymbol{I}$ stands for the identity operator.

Remark 2.5. Let $\left(\boldsymbol{u}_{H}, p_{H}\right):=\left(\boldsymbol{u}_{1}+\boldsymbol{u}_{e}, p_{0}+p_{e}\right)$. Then, (2.23) implies that

$$
\sigma\left(\boldsymbol{u}_{H}, \boldsymbol{v}_{1}\right)_{\Omega}-\left(p_{H}, \nabla \cdot \boldsymbol{v}_{1}\right)_{\Omega}=\left(\boldsymbol{f}, \boldsymbol{v}_{1}\right)_{\Omega} \quad \forall \boldsymbol{v}_{1} \in \mathbf{V}_{H},
$$

and hence, integrating by parts and using the fact that $\sigma \boldsymbol{u}_{H}+\nabla p_{H}=\boldsymbol{f}$ in each $K$, we see that $p_{H}$ satisfies

$$
\sum_{F \in \mathcal{E}_{H}}\left(\llbracket p_{H} \rrbracket, \boldsymbol{v}_{1} \cdot \boldsymbol{n}\right)_{F}=0 \quad \forall \boldsymbol{v}_{1} \in \mathbf{V}_{H} .
$$

Then, when enhanced with $p_{e}$, the discrete pressure is weakly continuous. On the other hand, since we also have $\llbracket \boldsymbol{u}_{H} \cdot \boldsymbol{n} \rrbracket=\mathbf{0}$ we see that $\llbracket \nabla p_{H} \cdot \boldsymbol{n} \rrbracket=0$ on the internal edges. Hence, our method naturally satisfies the conditions requested in [10] in the discontinuous Galerkin framework.

To complete the derivation we only have to notice that we can neglect the nonsymmetric term. This is ensured by the next lemma. As a matter of fact, the following result ensures us that the non-symmetric term is of a smaller size than the rest of the terms, and then in Lemma 3.9 below we will show that the solution of (2.23) and the solution of the symmetric method (2.27) below are superclose in the natural norms.

Lemma 2.6. There exists a positive constant $C$ such that

$$
\sum_{K \in \mathcal{T}_{H}}\left(\mathcal{D}_{K}^{u}\left(\llbracket q_{0} \rrbracket\right), \sigma \rho\left(\boldsymbol{v}_{1}\right)\right)_{K} \leq C\left\{\sum_{F \in \mathcal{E}_{H}} \tau_{F}\left\|\llbracket q_{0} \rrbracket\right\|_{0, F}^{2}\right\}^{\frac{1}{2}} \sqrt{\sigma \alpha} H\left\|\rho\left(\boldsymbol{v}_{1}\right)\right\|_{0, \Omega},
$$

for all $\boldsymbol{v}_{1} \in \mathbf{V}_{H}$ and $q_{0} \in Q_{H}$, where $\alpha:=\max \left\{\alpha_{F}: F \in \mathcal{E}_{H}\right\}$. 
Proof. Successively using the Cauchy-Schwarz inequality, (2.14), $\left\|\varphi_{F}\right\|_{0, K} \leq C H_{F}$, the definition of $\tau_{F}$ (cf. (2.20) ) and the mesh regularity we get

$$
\begin{aligned}
\sum_{K \in \mathcal{T}_{H}}\left(\mathcal{D}_{K}^{u}\left(\llbracket q_{0} \rrbracket\right), \sigma \rho\left(\boldsymbol{v}_{1}\right)\right)_{\Omega} & \leq \sum_{K \in \mathcal{T}_{H}}\left\|\mathcal{D}_{K}^{u}\left(\llbracket q_{0} \rrbracket\right)\right\|_{0, K} \sigma\left\|\rho\left(\boldsymbol{v}_{1}\right)\right\|_{0, K} \\
& \leq \sum_{K \in \mathcal{T}_{H}} \sum_{F \subseteq \partial K} \frac{\alpha_{F}}{\sigma} \int_{F}\left|\llbracket q_{0} \rrbracket\right|\left\|\boldsymbol{\varphi}_{F}\right\|_{0, K} \sigma\left\|\rho\left(\boldsymbol{v}_{1}\right)\right\|_{0, K} \\
& \leq C \sum_{K \in \mathcal{T}_{H}} \sum_{F \subseteq \partial K} \tau_{F} H_{F}^{\frac{1}{2}}\left\|\llbracket q_{0} \rrbracket\right\|_{0, F} \sigma\left\|\rho\left(\boldsymbol{v}_{1}\right)\right\|_{0, K} \\
& \leq C\left\{\sum_{F \in \mathcal{E}_{H}} \tau_{F}\left\|\llbracket q_{0} \rrbracket\right\|_{0, F}^{2}\right\}^{\frac{1}{2}} H \sqrt{\sigma \alpha}\left\|\rho\left(\boldsymbol{v}_{1}\right)\right\|_{0, \Omega},
\end{aligned}
$$

which ends the proof.

Hence, using this result we arrive at the following symmetric (and final) form of our Petrov-Galerkin Enriched Method: Find $\left(\boldsymbol{u}_{1}, p_{0}\right) \in \mathbf{V}_{H} \times Q_{H}$ such that

$$
\boldsymbol{B}\left(\left(\boldsymbol{u}_{1}, p_{0}\right),\left(\boldsymbol{v}_{1}, q_{0}\right)\right)=\mathbf{F}\left(\boldsymbol{v}_{1}, q_{0}\right),
$$

for all $\left(\boldsymbol{v}_{1}, q_{0}\right) \in \mathbf{V}_{H} \times Q_{H}$, where

$$
\begin{aligned}
\boldsymbol{B}\left(\left(\boldsymbol{u}_{1}, p_{0}\right),\left(\boldsymbol{v}_{1}, q_{0}\right)\right) & :=\mathbf{A}\left(\left(\rho\left(\boldsymbol{u}_{1}\right), p_{0}\right),\left(\rho\left(\boldsymbol{v}_{1}\right), q_{0}\right)\right)-\sum_{F \in \mathcal{E}_{H}} \tau_{F}\left(\llbracket p_{0} \rrbracket, \llbracket q_{0} \rrbracket\right)_{F}, \\
\mathbf{F}\left(\boldsymbol{v}_{1}, q_{0}\right) & :=\boldsymbol{L}\left(\boldsymbol{v}_{1}, q_{0}\right)-\sum_{K \in \mathcal{T}_{H}}\left(\mathcal{G}_{K}^{u}\left(g-\Pi_{K}(g)\right), \sigma \boldsymbol{v}_{1}\right)_{K},
\end{aligned}
$$

$\rho$ is the operator defined in (2.24), and the coefficient $\tau_{F}$ is defined in (2.20).

We end this section by presenting the local mass conservation result. The proof of this lemma is a direct application of the results from [6], $\S 2.3$, and hence we skip the details. We only stress here the fact that the techniques developed in this paper may be applied to any jump-based stabilized finite element method for the Darcy equation, and then, every low order method (e.g. the one from [15]) may be easily post-processed in order to get a locally conservative velocity field.

Lemma 2.7. Let $\boldsymbol{u}_{1}$ be the solution of (2.27) and $\boldsymbol{u}_{e}^{D}$ given by (2.14). Then

$$
\int_{K} \nabla \cdot\left(\boldsymbol{u}_{1}+\boldsymbol{u}_{e}^{D}\right)-g=0 \quad \forall K \in \mathcal{T}_{H} .
$$

\section{ERROR ANALYSIS OF THE SEMIDISCRETE PROBLEM}

In the sequel $C$ denotes a generic positive constant, independent of $H$ or $\sigma$, with values that may vary in each occurrence. From now on, and just for simplicity of the presentation, we will assume that $\alpha_{F}=\alpha$ for all $F \in \mathcal{E}_{H}$.

3.1. Preliminaries. We start by presenting the Clément interpolation operator (cf. [17, 23, 19]) $\mathcal{C}_{H}: H^{1}(\Omega) \rightarrow V_{H}$ (with the obvious extension to vector-valued functions), satisfying, for all $K \in \mathcal{T}_{H}$ and all $F \in \mathcal{E}_{H}$,

$$
\begin{aligned}
\left\|\mathcal{C}_{H}(v)\right\|_{1, \Omega} & \leq C_{c l e}\|v\|_{1, \Omega} \quad \forall v \in H^{1}(\Omega), \\
\left\|v-\mathcal{C}_{H}(v)\right\|_{m, K} & \leq C_{c l e} H_{K}^{t-m}|v|_{t, \omega_{K}} \quad \forall v \in H^{t}\left(\omega_{K}\right), \\
\left\|v-\mathcal{C}_{H}(v)\right\|_{0, F} & \leq C_{c l e} H_{F}^{t-\frac{1}{2}}|v|_{t, \omega_{F}} \quad \forall v \in H^{t}\left(\omega_{F}\right),
\end{aligned}
$$


for $t=1,2, m=0,1$, where $\omega_{K}=\left\{K^{\prime} \in \mathcal{T}_{H}: K \cap K^{\prime} \neq \emptyset\right\}$ and $\omega_{F}=\left\{K \in \mathcal{T}_{H}:\right.$ $K \cap F \neq \emptyset\}$.

We will also use the $L^{2}(\Omega)$ projection onto $Q_{H}$ which is denoted by $\Pi_{H}$ : $L^{2}(\Omega) \rightarrow Q_{H}$. This projection satisfies (cf. [19])

$$
\left\{\sum_{K \in \mathcal{T}_{H}}\left\|q-\Pi_{H}(q)\right\|_{m, K}^{2}\right\}^{\frac{1}{2}} \leq C H^{t-m}|q|_{t, \Omega} \quad \forall q \in H^{t}(\Omega) \cap L_{0}^{2}(\Omega),
$$

for $0 \leq m \leq t \leq 1$. Moreover, using (3.4) and the local trace inequality, i.e., there exists $C_{t}$ such that for all $K \in \mathcal{T}_{H}$ and all $v \in H^{1}(K)$,

$$
\|v\|_{0, \partial K}^{2} \leq C_{t}\left(H_{K}^{-1}\|v\|_{0, K}^{2}+H_{K}|v|_{1, K}^{2}\right)
$$

we obtain

$$
\left[\sum_{F \in \mathcal{E}_{H}} H_{F}\left\|\llbracket q-\Pi_{H}(q) \rrbracket\right\|_{0, F}^{2}\right]^{1 / 2} \leq C H|q|_{1, \Omega} .
$$

Before heading to stability, two auxiliary results are stated next.

Lemma 3.1. There exist two constants $C, C^{\prime}>0$ such that, for all $K \in \mathcal{T}_{H}$ and all $\boldsymbol{v}_{1} \in \mathbb{P}_{1}(K)^{2}$,

$$
C H_{K}^{\frac{1}{2}}\left\|\boldsymbol{v}_{1} \cdot \boldsymbol{n}\right\|_{0, \partial K} \leq\left\|\boldsymbol{v}_{1}\right\|_{0, K} \leq C^{\prime} H_{K}^{\frac{1}{2}}\left\|\boldsymbol{v}_{1} \cdot \boldsymbol{n}\right\|_{0, \partial K} .
$$

Proof. First, using (3.5) and an inverse inequality it follows that

$$
\left\|\boldsymbol{v}_{1} \cdot \boldsymbol{n}\right\|_{0, \partial K}^{2} \leq C_{t}\left(H_{K}^{-1}\left\|\boldsymbol{v}_{1}\right\|_{0, K}^{2}+H_{K}\left|\boldsymbol{v}_{1}\right|_{1, K}^{2}\right) \leq C H_{K}^{-1}\left\|\boldsymbol{v}_{1}\right\|_{0, K}^{2},
$$

and the first inequality follows. Now let $\hat{K}$ be the standard reference element of vertices $(0,0),(1,0)$ and $(0,1)$. Since in $\mathbb{P}_{1}(\hat{K})^{2}$ both quantities $\left\|\hat{\boldsymbol{w}}_{1}\right\|_{0, \hat{K}}$ and $\left\|\hat{\boldsymbol{w}}_{1} \cdot \boldsymbol{n}\right\|_{0, \partial \hat{K}}$ define norms, there exists $C>0$ such that

$$
\left\|\hat{\boldsymbol{w}}_{1}\right\|_{0, \hat{K}} \leq C\left\|\hat{\boldsymbol{w}}_{1} \cdot \hat{\boldsymbol{n}}\right\|_{0, \partial \hat{K}},
$$

for all $\hat{\boldsymbol{w}}_{1} \in \mathbb{P}_{1}(\hat{K})^{2}$. Now let $\hat{\boldsymbol{v}}_{1}$ be the Piola transform of $\boldsymbol{v}_{1}$ (cf. 11]). Using the definition of the Piola transform, (3.8) and the fact that $\boldsymbol{v}_{1} \cdot \boldsymbol{n}=H_{F}^{-1} \hat{\boldsymbol{v}}_{1} \cdot \hat{\boldsymbol{n}}$, we get

$$
\begin{aligned}
\left\|\boldsymbol{v}_{1}\right\|_{0, K}^{2} & \leq C\left\|\hat{\boldsymbol{v}}_{1}\right\|_{0, \hat{K}}^{2} \\
& \leq C\left\|\hat{\boldsymbol{v}}_{1} \cdot \hat{\boldsymbol{n}}\right\|_{0, \partial \hat{K}}^{2} \\
& =C \sum_{\hat{F} \subseteq \partial \hat{K}} \int_{\hat{F}}\left(\hat{\boldsymbol{v}}_{1} \cdot \hat{\boldsymbol{n}}\right)^{2} \\
& =C \sum_{F \subseteq \partial K} \int_{F} H_{F}^{-1} H_{F}^{2}\left(\boldsymbol{v}_{1} \cdot \boldsymbol{n}\right)^{2} \\
& \leq C H_{K}\left\|\boldsymbol{v}_{1} \cdot \boldsymbol{n}\right\|_{0, \partial K}^{2},
\end{aligned}
$$

and the result follows.

We now define the following mesh-dependent norm

$$
\|(\boldsymbol{v}, q)\|_{H}^{2}:=\sigma\|\boldsymbol{v}\|_{d i v, \Omega}^{2}+\frac{\alpha}{\sigma}\|q\|_{0, \Omega}^{2}+\sum_{F \in \mathcal{E}_{H}} \tau_{F}\|\llbracket q \rrbracket\|_{0, F}^{2},
$$

and we present the following result which will be fundamental in the proof of the inf-sup condition below. 
Lemma 3.2. There exists $C>0$ such that, for all $\left(\boldsymbol{v}_{1}, q_{0}\right) \in \mathbf{V}_{H} \times Q_{H}$,

$$
\left\|\left(\boldsymbol{v}_{1}, q_{0}\right)\right\|_{H} \leq C\left\|\left(\rho\left(\boldsymbol{v}_{1}\right), q_{0}\right)\right\|_{H} .
$$

Proof. First, using an inverse inequality, the mesh regularity, $\rho\left(\boldsymbol{v}_{1}\right) \cdot \boldsymbol{n}=\boldsymbol{v}_{1} \cdot \boldsymbol{n}$, and the fact that $\left\|\boldsymbol{v}_{1} \cdot \boldsymbol{n}\right\|_{-1 / 2, \partial K} \leq\left\|\boldsymbol{v}_{1}\right\|_{d i v, K}$ (cf. [23]), we obtain

$$
\begin{aligned}
\left\|\boldsymbol{v}_{1} \cdot \boldsymbol{n}\right\|_{0, \partial K}^{2} & =\sum_{F \subseteq \partial K}\left\|\boldsymbol{v}_{1} \cdot \boldsymbol{n}\right\|_{0, F}^{2} \\
& \leq C \sum_{F \subseteq \partial K} H_{F}^{-1}\left\|\boldsymbol{v}_{1} \cdot \boldsymbol{n}\right\|_{-1 / 2, F}^{2} \\
& \leq 3 C H_{K}^{-1}\left\|\boldsymbol{v}_{1} \cdot \boldsymbol{n}\right\|_{-1 / 2, \partial K}^{2} \\
& =C H_{K}^{-1}\left\|\rho\left(\boldsymbol{v}_{1}\right) \cdot \boldsymbol{n}\right\|_{-1 / 2, \partial K}^{2} \\
& \leq C H_{K}^{-1}\left(\left\|\rho\left(\boldsymbol{v}_{1}\right)\right\|_{0, K}^{2}+\left\|\nabla \cdot \boldsymbol{v}_{1}\right\|_{0, K}^{2}\right),
\end{aligned}
$$

and then

$$
H_{K}\left\|\boldsymbol{v}_{1} \cdot \boldsymbol{n}\right\|_{0, \partial K}^{2} \leq C\left(\left\|\rho\left(\boldsymbol{v}_{1}\right)\right\|_{0, K}^{2}+\left\|\nabla \cdot \boldsymbol{v}_{1}\right\|_{0, K}^{2}\right) .
$$

The result follows by applying Lemma 3.1. the definition of the norm $\|\cdot\|_{H}$ and the fact that $\mathcal{M}_{K}^{u}\left(\boldsymbol{v}_{1}\right)$ is a solenoidal function.

We end this section by proving some technical results involving the operators $\sigma \mathcal{M}_{K}^{u}$ and $\left.\rho\right|_{K}=\boldsymbol{I}-\sigma \mathcal{M}_{K}^{u}$.

Lemma 3.3. Let $K \in \mathcal{T}_{H}$. Then, for all $\boldsymbol{v} \in H^{1}(K)^{2}$ there holds

i) $(\rho(\boldsymbol{v}), \boldsymbol{v})_{K}=\|\rho(\boldsymbol{v})\|_{0, K}^{2}$;

ii) $\|\rho(\boldsymbol{v})\|_{0, K}^{2}=\|\boldsymbol{v}\|_{0, K}^{2}-\|\boldsymbol{v}-\rho(\boldsymbol{v})\|_{0, K}^{2}$;

iii) $\left\|\sigma \mathcal{M}_{K}^{u}(\boldsymbol{v})\right\|_{0, K} \leq\|\boldsymbol{v}\|_{0, K} ;\|\rho(\boldsymbol{v})\|_{0, K} \leq\|\boldsymbol{v}\|_{0, K}$;

iv) $\|\boldsymbol{v}-\rho(\boldsymbol{v})\|_{0, K} \leq \frac{H_{K}}{\pi}|\boldsymbol{v}|_{1, K}$.

Proof. The first three items follow directly from the fact that $\sigma \mathcal{M}_{K}^{u}$ (and hence, $\rho$ ) is an orthogonal projection with respect to the $L^{2}(K)^{2}$ inner product. To prove iv), let $\boldsymbol{v} \in H^{1}(K)^{2}$ and let us denote $\boldsymbol{v}_{0}=\Pi_{K}(\boldsymbol{v})$. Since $\boldsymbol{v}_{0}$ is a constant in each element, there holds that $\mathcal{M}_{K}^{u}\left(\boldsymbol{v}_{0}\right)=\mathbf{0}$, and then using iii) we arrive at

$$
\|\boldsymbol{v}-\rho(\boldsymbol{v})\|_{0, K}=\left\|\sigma \mathcal{M}_{K}^{u}(\boldsymbol{v})\right\|_{0, K}=\left\|\sigma \mathcal{M}_{K}^{u}\left(\boldsymbol{v}-\boldsymbol{v}_{0}\right)\right\|_{0, K} \leq\left\|\boldsymbol{v}-\boldsymbol{v}_{0}\right\|_{0, K} .
$$

Finally, in 25] the following optimal Poincaré inequality is proved:

$$
\inf _{c \in \mathbb{R}}\|v-c\|_{0, K} \leq \frac{H_{K}}{\pi}|v|_{1, K} \quad \forall v \in H^{1}(K),
$$

and iv) follows.

3.2. Stability and convergence. Before proving the stability we recall that, for all $q_{0} \in Q_{H}$ there exists (cf. [23]) $\boldsymbol{w} \in H_{0}^{1}(\Omega)^{2}$ such that

$$
\nabla \cdot \boldsymbol{w}=-\frac{1}{\sigma} q_{0} \quad \text { in } \Omega \quad \text { and } \quad\|\boldsymbol{w}\|_{1, \Omega} \leq \frac{C_{1}}{\sigma}\left\|q_{0}\right\|_{0, \Omega},
$$

where $C_{1}$ depends only on $\Omega$. 
Theorem 3.4. Let us suppose that $\alpha \leq \min \left\{\frac{1}{2 C_{t}}, \frac{3}{8}\right\}$. Then, there exists $\beta>0$, independent of $H, \sigma$ and $\alpha$, such that

$$
\sup _{\left(\boldsymbol{w}_{1}, t_{0}\right) \in \mathbf{V}_{H} \times Q_{H}-\{\mathbf{0}\}} \frac{\boldsymbol{B}\left(\left(\boldsymbol{v}_{1}, q_{0}\right),\left(\boldsymbol{w}_{1}, t_{0}\right)\right)}{\left\|\left(\boldsymbol{w}_{1}, t_{0}\right)\right\|_{H}} \geq \beta\left\|\left(\boldsymbol{v}_{1}, q_{0}\right)\right\|_{H},
$$

for all $\left(\boldsymbol{v}_{1}, q_{0}\right) \in \mathbf{V}_{H} \times Q_{H}$, and the problem (2.27) is well posed.

Proof. Let $\left(\boldsymbol{v}_{1}, q_{0}\right) \in \mathbf{V}_{H} \times Q_{H}$. Then, from the definition of $\boldsymbol{B}$ it follows that

$$
\boldsymbol{B}\left(\left(\boldsymbol{v}_{1}, q_{0}\right),\left(\boldsymbol{v}_{1},-q_{0}\right)\right)=\sigma\left\|\rho\left(\boldsymbol{v}_{1}\right)\right\|_{0, \Omega}^{2}+\sum_{F \in \mathcal{E}_{H}} \tau_{F}\left\|\llbracket q_{0} \rrbracket\right\|_{0, F}^{2} .
$$

Next, we see that since $\nabla \cdot \boldsymbol{v}_{1} \in Q_{H}$ for all $\boldsymbol{v}_{1} \in \mathbf{V}_{H}$, we can take $-\sigma \nabla \cdot \boldsymbol{v}_{1}$ as a test function leading to

$$
\begin{aligned}
& \boldsymbol{B}\left(\left(\boldsymbol{v}_{1}, q_{0}\right),\left(\mathbf{0},-\sigma \nabla \cdot \boldsymbol{v}_{1}\right)\right)=\sigma\left\|\nabla \cdot \boldsymbol{v}_{1}\right\|_{0, \Omega}^{2}+\sigma \sum_{F \in \mathcal{E}_{H}} \tau_{F}\left(\llbracket q_{0} \rrbracket, \llbracket \nabla \cdot \boldsymbol{v}_{1} \rrbracket\right)_{F} \\
& \quad \geq \sigma\left\|\nabla \cdot \boldsymbol{v}_{1}\right\|_{0, \Omega}^{2}-\frac{\sigma^{2}}{2} \sum_{F \in \mathcal{E}_{H}} \tau_{F}\left\|\llbracket \nabla \cdot \boldsymbol{v}_{1} \rrbracket\right\|_{0, F}^{2}-\frac{1}{2} \sum_{F \in \mathcal{E}_{H}} \tau_{F}\left\|\llbracket q_{0} \rrbracket\right\|_{0, F}^{2} .
\end{aligned}
$$

Using (3.5) we obtain

$$
\begin{aligned}
\sum_{F \in \mathcal{E}_{H}} \tau_{F}\left\|\llbracket \nabla \cdot \boldsymbol{v}_{1} \rrbracket\right\|_{0, F}^{2} & \leq \frac{2 \alpha}{\sigma} \sum_{K \in \mathcal{T}_{H}} H_{K}\left\|\nabla \cdot \boldsymbol{v}_{1}\right\|_{0, \partial K}^{2} \\
& \leq \frac{2 C_{t} \alpha}{\sigma} \sum_{K \in \mathcal{T}_{H}}\left\|\nabla \cdot \boldsymbol{v}_{1}\right\|_{0, K}^{2}=\frac{2 C_{t} \alpha}{\sigma}\left\|\nabla \cdot \boldsymbol{v}_{1}\right\|_{0, \Omega}^{2}
\end{aligned}
$$

and then (3.14) becomes

$$
\boldsymbol{B}\left(\left(\boldsymbol{v}_{1}, q_{0}\right),\left(\mathbf{0},-\sigma \nabla \cdot \boldsymbol{v}_{1}\right)\right) \geq \sigma\left(1-C_{t} \alpha\right)\left\|\nabla \cdot \boldsymbol{v}_{1}\right\|_{0, \Omega}^{2}-\frac{1}{2} \sum_{F \in \mathcal{E}_{H}} \tau_{F}\left\|\llbracket q_{0} \rrbracket\right\|_{0, F}^{2} .
$$

Now let $\boldsymbol{w} \in H_{0}^{1}(\Omega)^{2}$ be given by (3.12) and let $\boldsymbol{w}_{1}:=\mathcal{C}_{H}(\boldsymbol{w})$. Integrating by parts, using (3.3), the mesh regularity and (3.12), we arrive at

$$
\begin{aligned}
\frac{1}{\sigma}\left\|q_{0}\right\|_{0, \Omega}^{2} & =-\left(q_{0}, \nabla \cdot \boldsymbol{w}\right)_{\Omega} \\
& =-\left(q_{0}, \nabla \cdot\left(\boldsymbol{w}-\boldsymbol{w}_{1}\right)\right)_{\Omega}-\left(q_{0}, \nabla \cdot \boldsymbol{w}_{1}\right)_{\Omega} \\
& =-\sum_{F \in \mathcal{E}_{H}}\left(\llbracket q_{0} \rrbracket,\left(\boldsymbol{w}-\boldsymbol{w}_{1}\right) \cdot \boldsymbol{n}\right)_{F}-\left(q_{0}, \nabla \cdot \boldsymbol{w}_{1}\right)_{\Omega} \\
& \leq C_{c l e} \sum_{F \in \mathcal{E}_{H}} H_{F}^{\frac{1}{2}}\left\|\llbracket q_{0} \rrbracket\right\|_{0, F}|\boldsymbol{w}|_{1, \omega_{F}}-\left(q_{0}, \nabla \cdot \boldsymbol{w}_{1}\right)_{\Omega} \\
& \leq \sqrt{3} C_{c l e} C_{1}\left\{\sum_{F \in \mathcal{E}_{H}} \frac{H_{F}}{\sigma}\left\|\llbracket q_{0} \rrbracket\right\|_{0, F}^{2}\right\}^{\frac{1}{2}} \frac{\left\|q_{0}\right\|_{0, \Omega}}{\sqrt{\sigma}}-\left(q_{0}, \nabla \cdot \boldsymbol{w}_{1}\right)_{\Omega}
\end{aligned}
$$

and then

$$
-\left(q_{0}, \nabla \cdot \boldsymbol{w}_{1}\right)_{\Omega} \geq \frac{1}{2 \sigma}\left\|q_{0}\right\|_{0, \Omega}^{2}-\frac{C_{2}}{\alpha} \sum_{F \in \mathcal{E}_{H}} \tau_{F}\left\|\llbracket q_{0} \rrbracket\right\|_{0, F}^{2},
$$


where $C_{2}=\frac{3 C_{\text {cle }}^{2} C_{1}^{2}}{2}$. From Lemma 3.3 iii), (3.1) and (3.12), we then obtain

$$
\begin{aligned}
& \boldsymbol{B}\left(\left(\boldsymbol{v}_{1}, q_{0}\right),\left(\boldsymbol{w}_{1}, 0\right)\right)=\sigma\left(\rho\left(\boldsymbol{v}_{1}\right), \rho\left(\boldsymbol{w}_{1}\right)\right)_{\Omega}-\left(q_{0}, \nabla \cdot \boldsymbol{w}_{1}\right)_{\Omega} \\
& \geq-\gamma_{1}^{-1} \sigma\left\|\rho\left(\boldsymbol{v}_{1}\right)\right\|_{0, \Omega}^{2}-\sigma \gamma_{1}\left\|\rho\left(\boldsymbol{w}_{1}\right)\right\|_{0, \Omega}^{2} \\
&+\frac{1}{2 \sigma}\left\|q_{0}\right\|_{0, \Omega}^{2}-\frac{C_{2}}{\alpha} \sum_{F \in \mathcal{E}_{H}} \tau_{F}\left\|\llbracket q_{0} \rrbracket\right\|_{0, F}^{2} \\
& \geq \frac{1}{\sigma}\left(\frac{1}{2}-C_{1}^{2} C_{c l e}^{2} \gamma_{1}\right)\left\|q_{0}\right\|_{0, \Omega}^{2}-\gamma_{1}^{-1} \sigma\left\|\rho\left(\boldsymbol{v}_{1}\right)\right\|_{0, \Omega}^{2}-\frac{C_{2}}{\alpha} \sum_{F \in \mathcal{E}_{H}} \tau_{F}\left\|\llbracket q_{0} \rrbracket\right\|_{0, F}^{2} \\
&(3.17)= \frac{1}{4 \sigma}\left\|q_{0}\right\|_{0, \Omega}^{2}-4 C_{c l e}^{2} C_{1}^{2} \sigma\left\|\rho\left(\boldsymbol{v}_{1}\right)\right\|_{0, \Omega}^{2}-\frac{C_{2}}{\alpha} \sum_{F \in \mathcal{E}_{H}} \tau_{F}\left\|\llbracket q_{0} \rrbracket\right\|_{0, F}^{2},
\end{aligned}
$$

choosing $\gamma_{1}=\frac{1}{4 C_{c l e}^{2} C_{1}^{2}}$.

Finally, let $\left(\mathbf{z}_{1}, t_{0}\right):=\left(\boldsymbol{v}_{1},-q_{0}\right)+\delta_{1}\left(\mathbf{0},-\sigma \nabla \cdot \boldsymbol{v}_{1}\right)+\delta_{2}\left(\boldsymbol{w}_{1}, 0\right)$ with $\delta_{1}, \delta_{2}>0$. Then, collecting (3.13), (3.15) and (3.17), we obtain

$$
\begin{aligned}
& \boldsymbol{B}\left(\left(\boldsymbol{v}_{1}, q_{0}\right),\left(\mathbf{z}_{1}, t_{0}\right)\right) \geq \sigma\left\|\rho\left(\boldsymbol{v}_{1}\right)\right\|_{0, \Omega}^{2}+\sum_{F \in \mathcal{E}_{H}} \tau_{F}\left\|\llbracket q_{0} \rrbracket\right\|_{0, F}^{2} \\
&+\delta_{1} \sigma\left(1-C_{t} \alpha\right)\left\|\nabla \cdot \boldsymbol{v}_{1}\right\|_{0, \Omega}^{2}-\frac{\delta_{1}}{2} \sum_{F \in \mathcal{E}_{H}} \tau_{F}\left\|\llbracket q_{0} \rrbracket\right\|_{0, F}^{2} \\
&+\frac{\delta_{2}}{4 \sigma}\left\|q_{0}\right\|_{0, \Omega}^{2}-4 \delta_{2} C_{c l e}^{2} C_{1}^{2} \sigma\left\|\rho\left(\boldsymbol{v}_{1}\right)\right\|_{0, \Omega}^{2}-\frac{\delta_{2} C_{2}}{\alpha} \sum_{F \in \mathcal{E}_{H}} \tau_{F}\left\|\llbracket q_{0} \rrbracket\right\|_{0, F}^{2} \\
&= \sigma\left(1-4 \delta_{2} C_{c l e}^{2} C_{1}^{2}\right)\left\|\rho\left(\boldsymbol{v}_{1}\right)\right\|_{0, \Omega}^{2}+\delta_{1} \sigma\left(1-C_{t} \alpha\right)\left\|\nabla \cdot \boldsymbol{v}_{1}\right\|_{0, \Omega}^{2}+\frac{\delta_{2}}{4 \sigma}\left\|q_{0}\right\|_{0, \Omega}^{2} \\
&+\left(1-\frac{\delta_{1}}{2}-\frac{\delta_{2} C_{2}}{\alpha}\right) \sum_{F \in \mathcal{E}_{H}} \tau_{F}\left\|\llbracket q_{0} \rrbracket\right\|_{0, F}^{2} \\
&(3.18) \geq C\left\|\left(\rho\left(\boldsymbol{v}_{1}\right), q_{0}\right)\right\|_{H}^{2},
\end{aligned}
$$

if $\alpha \leq \frac{1}{2 C_{t}}, \delta_{1} \leq \frac{1}{2}$ and $\delta_{2}=\frac{\alpha}{2 C_{2}}$, thus guaranteeing that $C>0$ is independent of $\alpha$. The result then follows using Lemma 3.2 and the fact that, thanks to the choice of $\delta_{2},\left\|\left(\mathbf{z}_{1}, t_{0}\right)\right\|_{H} \leq C\left\|\left(\boldsymbol{v}_{1}, q_{0}\right)\right\|_{H}$, where $C$ does not depend on $\alpha$.

Remark 3.5. If we look carefully at the proof of the last result, we may see that, since the quantity $\left\|\rho\left(\boldsymbol{v}_{1}\right)\right\|_{0, \Omega}$ defines a norm in $\mathbf{V}_{H}$, the well-posedeness of (2.27) follows directly from (3.13), independent of the value of $\alpha$. The reason to prove an inf-sup condition is the control in the norm of the divergence, which, thanks to Lemma 3.2 , allows us to prove an error estimate for $\boldsymbol{u}-\boldsymbol{u}_{1}$, instead of $\boldsymbol{u}-\rho\left(\boldsymbol{u}_{1}\right)$, that would arise naturally from (3.13).

Next, we present the following consistency result.

Lemma 3.6. Let $(\boldsymbol{u}, p) \in H_{0}(\operatorname{div}, \Omega) \times\left[H^{1}(\Omega) \cap L_{0}^{2}(\Omega)\right]$ be the solution of (1.5) and let $\left(\boldsymbol{u}_{1}, p_{0}\right)$ be the solution of (2.27), respectively. Then

$$
\boldsymbol{B}\left(\left(\boldsymbol{u}-\boldsymbol{u}_{1}, p-p_{0}\right),\left(\boldsymbol{v}_{1}, q_{0}\right)\right) \leq C \sigma H|\boldsymbol{u}|_{1, \Omega}\left\|\boldsymbol{v}_{1}\right\|_{0, \Omega}
$$

for all $\left(\boldsymbol{v}_{1}, q_{0}\right) \in \mathbf{V}_{H} \times Q_{H}$. 
Proof. Noting that $\llbracket p \rrbracket=0$ a.e. across all the internal edges, using the definition of $\boldsymbol{B}, \mathbf{A}$ and Lemma 2.3, we easily see that

$$
\begin{aligned}
\boldsymbol{B} & \left((\boldsymbol{u}, p),\left(\boldsymbol{v}_{1}, q_{0}\right)\right)=\sigma\left(\rho(\boldsymbol{u}), \rho\left(\boldsymbol{v}_{1}\right)\right)_{\Omega}-\left(p, \nabla \cdot \boldsymbol{v}_{1}\right)_{\Omega}-\left(q_{0}, \nabla \cdot \boldsymbol{u}\right)_{\Omega} \\
= & \sigma \sum_{K \in \mathcal{T}_{H}}\left(\left(\boldsymbol{I}-\sigma \mathcal{M}_{K}^{u}\right)(\boldsymbol{u}),\left(\boldsymbol{I}-\sigma \mathcal{M}_{K}^{u}\right)\left(\boldsymbol{v}_{1}\right)\right)_{K}-\left(p, \nabla \cdot \boldsymbol{v}_{1}\right)_{\Omega}-\left(q_{0}, \nabla \cdot \boldsymbol{u}\right)_{\Omega} \\
= & \sigma\left(\boldsymbol{u}, \boldsymbol{v}_{1}\right)_{\Omega}-\sum_{K \in \mathcal{T}_{H}} \sigma\left(\sigma \mathcal{M}_{K}^{u}(\boldsymbol{u}), \boldsymbol{v}_{1}\right)_{K}-\left(p, \nabla \cdot \boldsymbol{v}_{1}\right)_{\Omega}-\left(q_{0}, \nabla \cdot \boldsymbol{u}\right)_{\Omega} \\
& +\sum_{K \in \mathcal{T}_{H}}\left(\mathcal{G}_{K}^{u}\left(\nabla \cdot \boldsymbol{u}-\Pi_{K}(\nabla \cdot \boldsymbol{u})-g+\Pi_{K}(g)\right), \sigma \boldsymbol{v}_{1}\right)_{K} \\
= & \mathbf{A}\left((\boldsymbol{u}, p),\left(\boldsymbol{v}_{1}, q_{0}\right)\right)-\sum_{K \in \mathcal{T}_{H}}\left(\mathcal{G}_{K}^{u}\left(g-\Pi_{K}(g)\right), \sigma \boldsymbol{v}_{1}\right)_{K} \\
& -\sum_{K \in \mathcal{T}_{H}} \sigma\left(\sigma \mathcal{M}_{K}^{u}(\boldsymbol{u}), \boldsymbol{v}_{1}\right)_{K}+\sum_{K \in \mathcal{T}_{H}}\left(\mathcal{G}_{K}^{u}\left(\nabla \cdot \boldsymbol{u}-\Pi_{K}(\nabla \cdot \boldsymbol{u})\right), \sigma \boldsymbol{v}_{1}\right)_{K} \\
= & \mathbf{F}\left(\boldsymbol{v}_{1}, q_{0}\right)-\sum_{K \in \mathcal{T}_{H}} \sigma\left(\sigma \mathcal{M}_{K}^{u}(\boldsymbol{u}), \boldsymbol{v}_{1}\right)_{K}+\sum_{K \in \mathcal{T}_{H}}\left(\mathcal{G}_{K}^{u}\left(\nabla \cdot \boldsymbol{u}-\Pi_{K}(\nabla \cdot \boldsymbol{u})\right), \sigma \boldsymbol{v}_{1}\right)_{K} \\
= & \boldsymbol{B}\left(\left(\boldsymbol{u}_{1}, p_{0}\right),\left(\boldsymbol{v}_{1}, q_{0}\right)\right)-\sum_{K \in \mathcal{T}_{H}} \sigma\left(\sigma \mathcal{M}_{K}^{u}(\boldsymbol{u}), \boldsymbol{v}_{1}\right)_{K} \\
& +\sum_{K \in \mathcal{T}_{H}}\left(\mathcal{G}_{K}^{u}\left(\nabla \cdot \boldsymbol{u}-\Pi_{K}(\nabla \cdot \boldsymbol{u})\right), \sigma \boldsymbol{v}_{1}\right)_{K},
\end{aligned}
$$

and then

$$
\begin{aligned}
& \boldsymbol{B}\left(\left(\boldsymbol{u}-\boldsymbol{u}_{1}, p-p_{0}\right),\left(\boldsymbol{v}_{1}, q_{0}\right)\right) \\
& \quad \leq \sum_{K \in \mathcal{T}_{H}}\left(\left\|\sigma \mathcal{M}_{K}^{u}(\boldsymbol{u})\right\|_{0, K}+\left\|\mathcal{G}_{K}^{u}\left(\nabla \cdot \boldsymbol{u}-\Pi_{K}(\nabla \cdot \boldsymbol{u})\right)\right\|_{0, K}\right) \sigma\left\|\boldsymbol{v}_{1}\right\|_{0, K}
\end{aligned}
$$

Next, to bound the term $\left\|\mathcal{G}_{K}^{u}\left(\nabla \cdot \boldsymbol{u}-\Pi_{K}(\nabla \cdot \boldsymbol{u})\right)\right\|_{0, K}$ we follow very closely the results from [6], Appendix A. First, we recall that, from (2.13), the problem satisfied by $\boldsymbol{w}:=\mathcal{G}_{K}^{u}\left(\nabla \cdot \boldsymbol{u}-\Pi_{K}(\nabla \cdot \boldsymbol{u})\right)$ is given by

$$
\begin{aligned}
& \sigma \boldsymbol{w}+\nabla \eta=\mathbf{0}, \quad \nabla \cdot \boldsymbol{w}=\nabla \cdot \boldsymbol{u}-\Pi_{K}(\nabla \cdot \boldsymbol{u}) \quad \text { in } K, \\
& \boldsymbol{w} \cdot \boldsymbol{n}=0 \text { on } \partial K \text {, }
\end{aligned}
$$

where $\eta \in L_{0}^{2}(K)$. Now, multiplying the first equation in (3.20) by $\boldsymbol{w}$, the second by $\eta$, adding both and integrating by parts we arrive at

$$
\sigma\|\boldsymbol{w}\|_{0, K}^{2}=\left(\nabla \cdot \boldsymbol{u}-\Pi_{K}(\nabla \cdot \boldsymbol{u}), \eta\right)_{K} \leq\left\|\nabla \cdot \boldsymbol{u}-\Pi_{K}(\nabla \cdot \boldsymbol{u})\right\|_{0, K}\|\eta\|_{0, K},
$$

and since $\eta \in L_{0}^{2}(K)$ then $\|\eta\|_{0, K} \leq \frac{H_{K}}{\pi}|\eta|_{1, K}=\sigma \frac{H_{K}}{\pi}\|\boldsymbol{w}\|_{0, K}$ and we get

$$
\sigma\|\boldsymbol{w}\|_{0, K}^{2} \leq \frac{H_{K}}{\pi}\left\|\nabla \cdot \boldsymbol{u}-\Pi_{K}(\nabla \cdot \boldsymbol{u})\right\|_{0, K}|\eta|_{1, K} \leq \sigma \frac{H_{K}}{\pi}\|\nabla \cdot \boldsymbol{u}\|_{0, K}\|\boldsymbol{w}\|_{0, K},
$$

which leads to

$$
\left\|\mathcal{G}_{K}^{u}\left(\nabla \cdot \boldsymbol{u}-\Pi_{K}(\nabla \cdot \boldsymbol{u})\right)\right\|_{0, K} \leq \frac{H_{K}}{\pi}\|\nabla \cdot \boldsymbol{u}\|_{0, K} .
$$

The result follows from (3.19) and Lemma 3.3 iv).

With this result in mind we can present the following convergence result. 
Theorem 3.7. Let us suppose that $(\boldsymbol{u}, p)$, the solution of (1.5), belongs to $H^{2}(\Omega)^{2} \times$ $H^{1}(\Omega)$, and let $\left(\boldsymbol{u}_{1}, p_{0}\right)$ be the solution of (2.27). Then, there exists $C>0$, independent of $H, \sigma$ and $\alpha$, such that

$$
\left\|\left(\boldsymbol{u}-\boldsymbol{u}_{1}, p-p_{0}\right)\right\|_{H} \leq C H\left(\sqrt{\frac{\sigma}{\alpha}}\|\boldsymbol{u}\|_{2, \Omega}+\frac{1}{\sqrt{\sigma}}|p|_{1, \Omega}\right) .
$$

Proof. First, let $\left(\boldsymbol{v}_{1}, q_{0}\right):=\left(\mathcal{C}_{H}(\boldsymbol{u}), \Pi_{H}(p)\right)$. Then, from Theorem 3.4 there exists $\left(\boldsymbol{w}_{1}, t_{0}\right) \in \mathbf{V}_{H} \times Q_{H}$ such that $\left\|\left(\boldsymbol{w}_{1}, t_{0}\right)\right\|_{H}=1$ and

$$
\beta\left\|\left(\boldsymbol{u}_{1}-\boldsymbol{v}_{1}, p_{0}-q_{0}\right)\right\|_{H} \leq \boldsymbol{B}\left(\left(\boldsymbol{u}_{1}-\boldsymbol{v}_{1}, p_{0}-q_{0}\right),\left(\boldsymbol{w}_{1}, t_{0}\right)\right) .
$$

Now, using Lemmas 3.2 and 3.6, the Cauchy-Schwarz inequality, the fact that $\left(p-q_{0}, \nabla \cdot \boldsymbol{w}_{1}\right)_{\Omega}=0$ and (3.2)-(3.4), we obtain

$$
\begin{aligned}
\beta \| & \left(\boldsymbol{u}_{1}-\boldsymbol{v}_{1}, p_{0}-q_{0}\right) \|_{H} \\
\leq & \boldsymbol{B}\left(\left(\boldsymbol{u}-\boldsymbol{v}_{1}, p-q_{0}\right),\left(\boldsymbol{w}_{1}, t_{0}\right)\right)+\boldsymbol{B}\left(\left(\boldsymbol{u}_{1}-\boldsymbol{u}, p_{0}-p\right),\left(\boldsymbol{w}_{1}, t_{0}\right)\right) \\
= & \sigma\left(\rho\left(\boldsymbol{u}-\boldsymbol{v}_{1}\right), \rho\left(\boldsymbol{w}_{1}\right)\right)_{\Omega}-\left(p-q_{0}, \nabla \cdot \boldsymbol{w}_{1}\right)_{\Omega}-\left(t_{0}, \nabla \cdot\left(\boldsymbol{u}-\boldsymbol{v}_{1}\right)\right)_{\Omega} \\
& -\sum_{F \in \mathcal{E}_{H}} \tau_{F}\left(\llbracket p-q_{0} \rrbracket, \llbracket t_{0} \rrbracket\right)_{F}+\boldsymbol{B}\left(\left(\boldsymbol{u}_{1}-\boldsymbol{u}, p_{0}-p\right),\left(\boldsymbol{w}_{1}, t_{0}\right)\right) \\
\leq & C\left\{\sigma\left\|\rho\left(\boldsymbol{u}-\boldsymbol{v}_{1}\right)\right\|_{0, \Omega}^{2}+\frac{\sigma}{\alpha}\left\|\nabla \cdot\left(\boldsymbol{u}-\boldsymbol{v}_{1}\right)\right\|_{0, \Omega}^{2}\right. \\
& \left.+\sum_{F \in \mathcal{E}_{H}} \tau_{F}\left\|\llbracket p-q_{0} \rrbracket\right\|_{0, F}^{2}+\sigma H^{2}|\boldsymbol{u}|_{1, \Omega}^{2}\right\}^{\frac{1}{2}} \\
& \times\left\{\sigma\left\|\rho\left(\boldsymbol{w}_{1}\right)\right\|_{0, \Omega}^{2}+\frac{\alpha}{\sigma}\left\|t_{0}\right\|_{0, \Omega}^{2}+\sum_{F \in \mathcal{E}_{H}} \tau_{F}\left\|\llbracket t_{0} \rrbracket\right\|_{0, F}^{2}+\sigma\left\|\boldsymbol{w}_{1}\right\|_{0, \Omega}^{2}\right\}^{\frac{1}{2}} \\
\leq & C\left\{\frac{\sigma}{\alpha}\left\|\boldsymbol{u}-\boldsymbol{v}_{1}\right\|_{d i v, \Omega}^{2}+\sum_{F \in \mathcal{E}_{H}} \tau_{F}\left\|\llbracket p-q_{0} \rrbracket\right\|_{0, F}^{2}+\sigma H^{2}|\boldsymbol{u}|_{1, \Omega}^{2}\right\}^{\frac{1}{2}} \\
\leq & C H\left(\sqrt{\frac{\sigma}{\alpha}}\|\boldsymbol{u}\|_{2, \Omega}+\frac{\alpha}{\sqrt{\sigma}}|p|_{1, \Omega}\right),
\end{aligned}
$$

and the result follows using the triangle inequality.

Next, as was mentioned in the previous section, in order to provide a mass conservative velocity field we must enhance $\boldsymbol{u}_{1}$ with the Raviart-Thomas field $\boldsymbol{u}_{e}^{D}$. The next result shows that this fact does not undermine the convergence of the method.

Corollary 3.8. Let $(\boldsymbol{u}, p)$ and $\left(\boldsymbol{u}_{1}, p_{0}\right)$ be the solutions of (1.5) and (2.27), respectively. Then, under the hypothesis of the previous theorem there exists $C$ such that

$$
\begin{aligned}
& \left\|\boldsymbol{u}-\boldsymbol{u}_{1}-\boldsymbol{u}_{e}^{D}\right\|_{d i v, \Omega} \leq C H\left(\frac{1}{\sqrt{\alpha}}\|\boldsymbol{u}\|_{2, \Omega}+\frac{1}{\sigma}|p|_{1, \Omega}\right) \\
& \left\|\boldsymbol{u}-\boldsymbol{u}_{1}-\boldsymbol{u}_{e}^{M}-\boldsymbol{u}_{e}^{D}\right\|_{d i v, \Omega} \leq C H\left(\frac{1}{\sqrt{\alpha}}\|\boldsymbol{u}\|_{2, \Omega}+\frac{1}{\sigma}|p|_{1, \Omega}\right),
\end{aligned}
$$

where $\boldsymbol{u}_{e}^{D}$ is given by (2.14) and $\left.\boldsymbol{u}_{e}^{M}\right|_{K}=-\sigma \mathcal{M}_{K}^{u}\left(\boldsymbol{u}_{1}\right)$. 
Proof. We use the local mass conservation feature to prove (3.21). In fact, from Lemma 2.7 we obtain that

$$
\int_{K} \nabla \cdot\left(\boldsymbol{u}_{1}+\boldsymbol{u}_{e}^{D}\right)=\int_{K} g \quad \forall K \in \mathcal{T}_{H},
$$

and then $\left.\nabla \cdot\left(\boldsymbol{u}_{1}+\boldsymbol{u}_{e}^{D}\right)\right|_{K}=\Pi_{K}(g)$ in each $K$, which leads to

$$
\left\|\nabla \cdot\left(\boldsymbol{u}-\boldsymbol{u}_{1}-\boldsymbol{u}_{e}^{D}\right)\right\|_{0, K}=\left\|g-\Pi_{K}(g)\right\|_{0, K} \leq \frac{H_{K}}{\pi}|\nabla \cdot \boldsymbol{u}|_{1, K} .
$$

Following the same arguments from the proof of Lemma 2.6 we can prove that

$$
\left\|\boldsymbol{u}_{e}^{D}\right\|_{0, \Omega} \leq C \sigma^{-\frac{1}{2}} H\left\{\sum_{F \in \mathcal{E}_{H}} \tau_{F}\left\|\llbracket p-p_{0} \rrbracket\right\|_{0, F}^{2}\right\}^{\frac{1}{2}},
$$

and then using the previous theorem we obtain

$$
\left\|\boldsymbol{u}-\boldsymbol{u}_{1}-\boldsymbol{u}_{e}^{D}\right\|_{0, \Omega} \leq C H\left(\frac{1}{\sqrt{\alpha}}\|\boldsymbol{u}\|_{2, \Omega}+\frac{1}{\sigma}|p|_{1, \Omega}\right),
$$

and (3.21) follows from (3.24), 3.26) and Theorem 3.7

Next, to prove (3.22) we recall that $\boldsymbol{u}_{e}^{M}=-\sigma \mathcal{M}_{K}^{u}\left(\boldsymbol{u}_{1}\right)=-\boldsymbol{u}_{1}+\rho\left(\boldsymbol{u}_{1}\right)$, and then using (3.25) and Lemma 3.3.

$$
\begin{aligned}
\left\|\boldsymbol{u}-\rho\left(\boldsymbol{u}_{1}\right)-\boldsymbol{u}_{e}^{D}\right\|_{0, \Omega} & \leq\|\boldsymbol{u}-\rho(\boldsymbol{u})\|_{0, \Omega}+\left\|\rho(\boldsymbol{u})-\rho\left(\boldsymbol{u}_{1}\right)\right\|_{0, \Omega}+\left\|\boldsymbol{u}_{e}^{D}\right\|_{0, \Omega} \\
& \leq C H|\boldsymbol{u}|_{1, \Omega}+\left\|\boldsymbol{u}-\boldsymbol{u}_{1}\right\|_{0, \Omega}+C \frac{H}{\sqrt{\sigma}}\left\|\left(\boldsymbol{u}-\boldsymbol{u}_{1}, p-p_{0}\right)\right\|_{H},
\end{aligned}
$$

and the result follows using the previous theorem and the fact that $\boldsymbol{u}_{e}^{M}$ is solenoidal.

We end this section by explaining more in depth why we can actually neglect the non-symmetric term.

Lemma 3.9. Let us suppose that $\alpha$ is small enough. Then, there exists $\beta_{1}>0$, independent of $H$ and $\alpha$, such that

$$
\beta_{1}\left\|\left(\boldsymbol{v}_{1}, q_{0}\right)\right\|_{H} \leq \sup _{\left(\boldsymbol{w}_{1}, t_{0}\right) \in \mathbf{V}_{H} \times Q_{H}-\{\mathbf{0}\}} \frac{\boldsymbol{B}_{n s}\left(\left(\boldsymbol{v}_{1}, q_{0}\right),\left(\boldsymbol{w}_{1}, t_{0}\right)\right)}{\left\|\left(\boldsymbol{w}_{1}, t_{0}\right)\right\|_{H}},
$$

for all $\left(\boldsymbol{v}_{1}, q_{0}\right) \in \mathbf{V}_{H} \times Q_{H}$. Furthermore, there exists $C>0$ independent of $H, \sigma$ and $\alpha$ such that

$$
\left\|\left(\boldsymbol{u}_{1}-\hat{\boldsymbol{u}}_{1}, p_{0}-\hat{p}_{0}\right)\right\|_{H} \leq C H^{2}\left(\sqrt{\sigma}|\boldsymbol{u}|_{2, \Omega}+\frac{1}{\sqrt{\sigma}}|p|_{1, \Omega}\right),
$$

where $\left(\boldsymbol{u}_{1}, p_{0}\right)$ and $\left(\hat{\boldsymbol{u}}_{1}, \hat{p}_{0}\right)$ are the solutions of (2.27) and (2.23), respectively. 
Proof. For the inf-sup condition we start noting that, from the definition of $\boldsymbol{B}_{n s}$ and Lemma 2.6 there follows that

$$
\begin{aligned}
\boldsymbol{B}_{n s}\left(\left(\boldsymbol{v}_{1}, q_{0}\right),\left(\boldsymbol{v}_{1},-q_{0}\right)\right)= & \sigma\left\|\rho\left(\boldsymbol{v}_{1}\right)\right\|_{0, K}^{2} \\
& +\sum_{K \in \mathcal{T}_{H}}\left(\mathcal{D}_{K}^{u}\left(\llbracket q_{0} \rrbracket\right), \rho\left(\boldsymbol{v}_{1}\right)\right)_{K}+\sum_{F \in \mathcal{E}_{H}} \tau_{F}\left\|\llbracket q_{0} \rrbracket\right\|_{0, F}^{2} \\
\geq & \frac{\sigma}{2}\left(1-\alpha C_{*}^{2} H^{2}\right)\left\|\rho\left(\boldsymbol{v}_{1}\right)\right\|_{0, K}^{2}+\frac{1}{2} \sum_{F \in \mathcal{E}_{H}} \tau_{F}\left\|\llbracket q_{0} \rrbracket\right\|_{0, F}^{2} \\
\geq & \frac{3 \sigma}{8}\left\|\rho\left(\boldsymbol{v}_{1}\right)\right\|_{0, K}^{2}+\frac{1}{2} \sum_{F \in \mathcal{E}_{H}} \tau_{F}\left\|\llbracket q_{0} \rrbracket\right\|_{0, F}^{2},
\end{aligned}
$$

if we suppose that $\alpha$ satisfies $\alpha \leq \frac{1}{4 C_{*}^{2} H^{2}}$, where $C_{*}$ is the constant from Lemma 2.6. The remaining part of the proof of the inf-sup condition is completely analogous to the proof of Theorem 3.4, and therefore we skip the details. To prove the error estimate we see that from the inf-sup condition and using the definition of $\boldsymbol{B}$ and $\boldsymbol{B}_{n s}$ and Lemma 2.6. we have

$$
\begin{aligned}
\beta_{1}\left\|\left(\boldsymbol{u}_{1}-\hat{\boldsymbol{u}}_{1}, p_{0}-\hat{p}_{0}\right)\right\|_{H} & \leq \sup _{\left(\boldsymbol{w}_{1}, t_{0}\right) \in \mathbf{V}_{H} \times Q_{H}-\{\mathbf{0}\}} \frac{\boldsymbol{B}_{n s}\left(\left(\boldsymbol{u}_{1}-\hat{\boldsymbol{u}}_{1}, p_{0}-\hat{p}_{0}\right),\left(\boldsymbol{w}_{1}, t_{0}\right)\right)}{\left\|\left(\boldsymbol{w}_{1}, t_{0}\right)\right\|_{H}} \\
& =\sup _{\left(\boldsymbol{w}_{1}, t_{0}\right) \in \mathbf{V}_{H} \times Q_{H}-\{\mathbf{0}\}} \frac{\boldsymbol{B}_{n s}\left(\left(\boldsymbol{u}_{1}, p_{0}\right),\left(\boldsymbol{w}_{1}, t_{0}\right)\right)-\mathbf{F}\left(\boldsymbol{w}_{1}, t_{0}\right)}{\left\|\left(\boldsymbol{w}_{1}, t_{0}\right)\right\|_{H}} \\
& =\sup _{\left(\boldsymbol{w}_{1}, t_{0}\right) \in \mathbf{V}_{H} \times Q_{H}-\{\mathbf{0}\}} \frac{\sum_{K \in \mathcal{T}_{H}}\left(\mathcal{D}_{K}^{u}\left(\llbracket p_{0} \rrbracket\right), \sigma \boldsymbol{w}_{1}\right)_{K}}{\left\|\left(\boldsymbol{w}_{1}, t_{0}\right)\right\|_{H}} \\
& \leq \sqrt{\alpha} C H\left\{\sum_{F \in \mathcal{E}_{H}} \tau_{F}\left\|\llbracket p-p_{0} \rrbracket\right\|_{0, F}^{2}\right\}^{\frac{1}{2}} \\
& \leq \sqrt{\alpha} C H\left\|\left(\boldsymbol{u}-\boldsymbol{u}_{1}, p-p_{0}\right)\right\|_{H},
\end{aligned}
$$

and the result follows using Theorem 3.7

\section{A two-Level Finite Element method}

First we start by remarking that, from the definition of $\mathcal{M}_{K}$ and $\mathcal{G}_{K}$ (cf. (2.11)(2.13)), it follows that

$$
\begin{gathered}
\rho\left(\boldsymbol{v}_{1}\right)=\boldsymbol{v}_{1}-\sigma \mathcal{M}_{K}^{u}\left(\boldsymbol{v}_{1}\right)=\nabla \mathcal{M}_{K}^{p}\left(\boldsymbol{v}_{1}\right), \\
\sigma \mathcal{G}_{K}^{u}\left(g-\Pi_{K}(g)\right)=-\nabla \mathcal{G}_{K}^{p}\left(g-\Pi_{K}(g)\right),
\end{gathered}
$$

and then the method (2.27) may be rewritten in the following equivalent way:

$$
\begin{aligned}
& \sum_{K \in \mathcal{T}_{H}} \sigma\left(\nabla \mathcal{M}_{K}^{p}\left(\boldsymbol{u}_{1}\right), \nabla \mathcal{M}_{K}^{p}\left(\boldsymbol{v}_{1}\right)\right)_{K}-\left(p_{0}, \nabla \cdot \boldsymbol{v}_{1}\right)_{\Omega} \\
& \quad-\left(q_{0}, \nabla \cdot \boldsymbol{u}_{1}\right)_{\Omega}-\sum_{F \in \mathcal{E}_{H}} \tau_{F}\left(\llbracket p_{0} \rrbracket, \llbracket q_{0} \rrbracket\right)_{F} \\
& \quad=\left(\boldsymbol{f}, \boldsymbol{v}_{1}\right)_{\Omega}-\left(g, q_{0}\right)_{\Omega}+\sum_{K \in \mathcal{T}_{H}}\left(\nabla \mathcal{G}_{K}^{p}\left(g-\Pi_{K}(g)\right), \boldsymbol{v}_{1}\right)_{K},
\end{aligned}
$$

for all $\left(\boldsymbol{v}_{1}, q_{0}\right) \in \mathbf{V}_{H} \times Q_{H}$. Let us further remark that, from (2.8) and (2.10), the functions $p_{e}^{M}\left(\boldsymbol{v}_{1}\right)=\mathcal{M}_{K}^{p}\left(\boldsymbol{v}_{1}\right)$ and $p_{e}^{g}=\mathcal{G}_{K}^{p}\left(g-\Pi_{K}(g)\right)$ may be computed by 
solving the following Neumann problems in each $K \in \mathcal{T}_{H}$ :

$$
\begin{aligned}
-\Delta p_{e}^{M}\left(\boldsymbol{v}_{1}\right) & =-\nabla \cdot \boldsymbol{v}_{1} \quad \text { in } K, \\
\partial_{\boldsymbol{n}} p_{e}^{M}\left(\boldsymbol{v}_{1}\right) & =\boldsymbol{v}_{1} \cdot \boldsymbol{n} \quad \text { on } \partial K,
\end{aligned}
$$

and

$$
\begin{aligned}
-\Delta p_{e}^{g} & =\sigma\left(g-\Pi_{K}(g)\right) \quad \text { in } K, \\
\partial_{\boldsymbol{n}} p_{e}^{g} & =0 \quad \text { on } \partial K,
\end{aligned}
$$

respectively. Using these writings for the local problems, a two-level finite element method arises by replacing $p_{e}^{M}$ and $p_{e}^{g}$ in (4.2) by suitable finite element approximations. To do this, let, for each $K \in \mathcal{T}_{H},\left\{\mathcal{T}_{h}^{K}\right\}_{h>0}$ be a regular family of triangulations of $K$ built using triangles $\bar{K} \subseteq K$ with diameter less than or equal to $h$ (the value for $h$ may vary from one element to another, but for simplicity of the presentation it will always be denoted by $h$ ), and let

$$
\mathcal{R}_{h}^{K}:=\left\{\xi_{h} \in \mathcal{C}^{0}(K):\left.\xi_{h}\right|_{\bar{K}} \in \mathbb{P}_{l}(\bar{K}), \forall \bar{K} \in \mathcal{T}_{h}^{K}\right\} \cap L_{0}^{2}(K),
$$

where $l \geq 1$. Hence, we propose the following discretizations for (4.3) and (4.4): Find $p_{h}\left(\boldsymbol{v}_{1}\right) \in \mathcal{R}_{h}^{K}$ such that

$$
\int_{K} \nabla p_{h}\left(\boldsymbol{v}_{1}\right) \cdot \nabla \xi_{h}=\int_{K} \boldsymbol{v}_{1} \cdot \nabla \xi_{h} \quad \forall \xi_{h} \in \mathcal{R}_{h}^{K},
$$

and: Find $p_{h}^{g} \in \mathcal{R}_{h}^{K}$ such that

$$
\int_{K} \nabla p_{h}^{g} \cdot \nabla \xi_{h}=\sigma \int_{K}\left(g-\Pi_{K}(g)\right) \xi_{h} \quad \forall \xi_{h} \in \mathcal{R}_{h}^{K},
$$

respectively. With these approximations we introduce our two-level finite element method: Find $\left(\boldsymbol{u}_{1, h}, p_{0, h}\right) \in \mathbf{V}_{H} \times Q_{H}$ such that:

$$
\boldsymbol{B}_{h}\left(\left(\boldsymbol{u}_{1, h}, p_{0, h}\right),\left(\boldsymbol{v}_{1}, q_{0}\right)\right)=\mathbf{F}_{h}\left(\boldsymbol{v}_{1}, q_{0}\right) \quad \forall\left(\boldsymbol{v}_{1}, q_{0}\right) \in \mathbf{V}_{H} \times Q_{H},
$$

where

$$
\begin{aligned}
\boldsymbol{B}_{h}\left(\left(\boldsymbol{v}_{1}, q_{0}\right),\left(\boldsymbol{w}_{1}, t_{0}\right)\right):= & \sum_{K \in \mathcal{T}_{H}} \sigma\left(\nabla p_{h}\left(\boldsymbol{v}_{1}\right), \nabla p_{h}\left(\boldsymbol{w}_{1}\right)\right)_{K}-\left(q_{0}, \nabla \cdot \boldsymbol{w}_{1}\right)_{\Omega} \\
& -\left(t_{0}, \nabla \cdot \boldsymbol{v}_{1}\right)_{\Omega}-\sum_{F \in \mathcal{E}_{H}} \tau_{F}\left(\llbracket q_{0} \rrbracket, \llbracket t_{0} \rrbracket\right)_{F}
\end{aligned}
$$

and

$$
\mathbf{F}_{h}\left(\boldsymbol{v}_{1}, q_{0}\right):=\left(\boldsymbol{f}, \boldsymbol{v}_{1}\right)_{\Omega}-\left(g, q_{0}\right)_{\Omega}+\sum_{K \in \mathcal{T}_{H}}\left(\nabla p_{h}^{g}, \boldsymbol{v}_{1}\right)_{K},
$$

respectively.

4.1. Numerical analysis of the fully discrete method. To prove stability we start by proving the following lemma.

Lemma 4.1. There exists $C>0$, independent of $H, h, \sigma$ and $K$, such that

$$
\begin{aligned}
\left|p_{e}^{M}(\boldsymbol{v})\right|_{1, K} & \leq\|\boldsymbol{v}\|_{0, K}, \\
\left|p_{e}^{M}(\boldsymbol{v})-p_{h}(\boldsymbol{v})\right|_{1, K} & \leq C h|\boldsymbol{v}|_{1, K}, \\
\left|p_{e}^{g}-p_{h}^{g}\right|_{1, K} & \leq \frac{C h H_{K}^{t}}{\sigma}|g|_{t, K},
\end{aligned}
$$

for all $\boldsymbol{v} \in H^{1}(K)^{2}$, and for $t=0,1$. 
Proof. For the first estimate we consider $\xi_{h}=p_{h}(\boldsymbol{v})$ in (4.6) and apply the CauchySchwarz inequality to prove that $\left|p_{h}(\boldsymbol{v})\right|_{1, K}^{2} \leq\|\boldsymbol{v}\|_{0, K}\left|p_{h}(\boldsymbol{v})\right|_{1, K}$.

For the remaining parts, we start by stating the following result from [23], Theorem 3.9 , p. 55 (which is also valid in two space dimensions): If $\boldsymbol{w} \in H_{0}(\operatorname{div}, K) \cap$ $H(\operatorname{curl}, K)$, then $\boldsymbol{w} \in H^{1}(K)^{2}$ and satisfies

$$
|\boldsymbol{w}|_{1, K} \leq\|\nabla \cdot \boldsymbol{w}\|_{0, K}+\|\operatorname{curl}(\boldsymbol{w})\|_{0, K} .
$$

Then, recalling the mixed form of the problem satisfied by $p_{e}^{M}(\boldsymbol{v})$, i.e.,

$$
\begin{gathered}
\sigma \boldsymbol{u}_{e}^{M}(\boldsymbol{v})+\nabla p_{e}^{M}(\boldsymbol{v})=\boldsymbol{v}, \quad \nabla \cdot \boldsymbol{u}_{e}^{M}(\boldsymbol{v})=0 \quad \text { in } K, \\
\boldsymbol{u}_{e}^{M}(\boldsymbol{v}) \cdot \boldsymbol{n}=0 \quad \text { on } \partial K,
\end{gathered}
$$

and taking the curl from the first equation in (4.15) we see that $\boldsymbol{u}_{e}^{M}(\boldsymbol{v}) \in H_{0}(\operatorname{div}, K)$ $\cap H($ curl, $K)$, which implies $\boldsymbol{u}_{e}^{M}(\boldsymbol{v}) \in H^{1}(K)^{2}$ and satisfies (4.14). From (4.15) we then see that $p_{e}^{M}(\boldsymbol{v}) \in H^{2}(K)$ and satisfies

$$
\left|p_{e}^{M}(\boldsymbol{v})\right|_{2, K} \leq \sigma\left|\boldsymbol{u}_{e}^{M}(\boldsymbol{v})\right|_{1, K}+|\boldsymbol{v}|_{1, K} \leq\|\operatorname{curl}(\boldsymbol{v})\|_{0, K}+|\boldsymbol{v}|_{1, K}
$$

where we have also used the fact that $\left\|\operatorname{curl}\left(\boldsymbol{u}_{e}^{M}(\boldsymbol{v})\right)\right\|_{0, K} \leq \sigma^{-1}\|\operatorname{curl}(\boldsymbol{v})\|_{0, K}$. This leads to

$$
\left|p_{e}^{M}(\boldsymbol{v})-p_{h}(\boldsymbol{v})\right|_{1, K} \leq C h\left|p_{e}^{M}(\boldsymbol{v})\right|_{2, K} \leq C h|\boldsymbol{v}|_{1, K},
$$

where we have used the fact that $l \geq 1$ and standard finite element estimates (see, e.g., [19]). Following analogous steps we can prove (4.13).

The next result may be seen as a fully discrete version of Lemma 3.2 .

Lemma 4.2. Let $\|\cdot\|_{h}$ be the mesh-dependent norm given by

$\left\|\left(\boldsymbol{v}_{1}, q_{0}\right)\right\|_{h}^{2}:=\sum_{K \in \mathcal{T}_{H}} \sigma\left\|\nabla p_{h}\left(\boldsymbol{v}_{1}\right)\right\|_{0, K}^{2}+\sigma\left\|\nabla \cdot \boldsymbol{v}_{1}\right\|_{0, \Omega}^{2}+\frac{\alpha}{\sigma}\left\|q_{0}\right\|_{0, \Omega}^{2}+\sum_{F \in \mathcal{E}_{H}} \tau_{F}\left\|\llbracket q_{0} \rrbracket\right\|_{0, F}^{2}$,

and let us suppose that there exists $C_{0}>0$ such that $h \leq C_{0} H_{K}$, for all $K \in \mathcal{T}_{H}$. Then, there exists $C>0$ independent of $H, h, \sigma$ or $\alpha$ such that

$$
\left\|\left(\boldsymbol{v}_{1}, q_{0}\right)\right\|_{H} \leq C\left\|\left(\boldsymbol{v}_{1}, q_{0}\right)\right\|_{h},
$$

for all $\left(\boldsymbol{v}_{1}, q_{0}\right) \in \mathbf{V}_{H} \times Q_{H}$.

Proof. From Lemma 3.2 we know that there exists $C>0$ such that

$$
\begin{aligned}
& \left\|\left(\boldsymbol{v}_{1}, q_{0}\right)\right\|_{H}^{2} \leq C\left\|\left(\rho\left(\boldsymbol{v}_{1}\right), q_{0}\right)\right\|_{H}^{2} \\
& =C\left\{\sum_{K \in \mathcal{T}_{H}} \sigma\left\|\nabla p_{e}^{M}\left(\boldsymbol{v}_{1}\right)\right\|_{0, K}^{2}+\sigma\left\|\nabla \cdot \boldsymbol{v}_{1}\right\|_{0, \Omega}^{2}+\frac{\alpha}{\sigma}\left\|q_{0}\right\|_{0, \Omega}^{2}+\sum_{F \in \mathcal{E}_{H}} \tau_{F}\left\|\llbracket q_{0} \rrbracket\right\|_{0, F}^{2}\right\}
\end{aligned}
$$

$$
\leq C\left\{\sum_{K \in \mathcal{T}_{H}} \sigma\left|p_{e}^{M}\left(\boldsymbol{v}_{1}\right)-p_{h}\left(\boldsymbol{v}_{1}\right)\right|_{1, K}^{2}+\left\|\left(\boldsymbol{v}_{1}, q_{0}\right)\right\|_{h}^{2}\right\} .
$$

Now, using Lemma 4.1 and an inverse inequality we obtain

$$
\left|p_{e}^{M}\left(\boldsymbol{v}_{1}\right)-p_{h}\left(\boldsymbol{v}_{1}\right)\right|_{1, K}^{2} \leq C h^{2}\left|p_{e}^{M}\left(\boldsymbol{v}_{1}\right)\right|_{2, K}^{2} \leq C h^{2}\left|\boldsymbol{v}_{1}\right|_{1, K}^{2} \leq C h^{2} H_{K}^{-2}\left\|\boldsymbol{v}_{1}\right\|_{0, K}^{2},
$$

and then (4.18) becomes

$$
\left\|\left(\boldsymbol{v}_{1}, q_{0}\right)\right\|_{H}^{2} \leq C \sigma \sum_{K \in \mathcal{T}_{H}} h^{2} H_{K}^{-2}\left\|\boldsymbol{v}_{1}\right\|_{0, K}^{2}+C\left\|\left(\boldsymbol{v}_{1}, q_{0}\right)\right\|_{h}^{2},
$$

and the result follows supposing that $h \leq \frac{H_{K}}{\sqrt{2 C}}$. 
Remark 4.3. As will be clear after the following lemma, the last result shows us, in particular, that it is enough to choose in advance one type of mesh to solve the local problems in each element, without the need to refine the subgrid mesh if the coarse mesh is refined, and independently of having a coarse mesh with very different sizes. Hence, the computation of $p_{h}\left(\boldsymbol{v}_{1}\right)$ has the same cost over all the elements, and it can indeed be inexpensive.

Lemma 4.4. Under the hypothesis of the previous lemma, there exists $\beta_{2}>0$ independent of $H, h$ and $\alpha$ such that

$$
\sup _{\left(\boldsymbol{w}_{1}, t_{0}\right) \in \mathbf{V}_{H} \times Q_{H}} \frac{\boldsymbol{B}_{h}\left(\left(\boldsymbol{v}_{1}, q_{0}\right),\left(\boldsymbol{w}_{1}, t_{0}\right)\right)}{\left\|\left(\boldsymbol{w}_{1}, t_{0}\right)\right\|_{H}} \geq \beta_{2}\left\|\left(\boldsymbol{v}_{1}, q_{0}\right)\right\|_{H},
$$

for all $\left(\boldsymbol{v}_{1}, q_{0}\right) \in \mathbf{V}_{H} \times Q_{H}$.

Proof. Let $\left(\boldsymbol{v}_{1}, q_{0}\right) \in \mathbf{V}_{H} \times Q_{H}$. Following exactly the same arguments from Theorem 3.4 (this time using (4.11) in (3.17)), we can build $\left(\mathbf{z}_{1}, t_{0}\right) \in \mathbf{V}_{H} \times Q_{H}$ such that $\left\|\left(\mathbf{z}_{1}, t_{0}\right)\right\|_{h} \leq C\left\|\left(\boldsymbol{v}_{1}, q_{0}\right)\right\|_{h}$ with $C$ independent of $H, h, \sigma$ and $\alpha$, and such that

$$
\boldsymbol{B}_{h}\left(\left(\boldsymbol{v}_{1}, q_{0}\right),\left(\mathbf{z}_{1}, t_{0}\right)\right) \geq C\left\|\left(\boldsymbol{v}_{1}, q_{0}\right)\right\|_{h}^{2},
$$

and the result follows from Lemma 4.2 .

We end this section by proving the main error estimate for the method (4.8).

Theorem 4.5. Under all the previous hypothesis, there exists $C>0$ independent of $H, h, \sigma$ and $\alpha$ such that

$$
\left\|\left(\boldsymbol{u}-\boldsymbol{u}_{1, h}, p-p_{0, h}\right)\right\|_{H} \leq C\left(\frac{h H^{t}}{\sqrt{\sigma}}|g|_{t, \Omega}+\sqrt{\frac{\sigma}{\alpha}}(H+h)\|\boldsymbol{u}\|_{2, \Omega}+\frac{H}{\sqrt{\sigma}}|p|_{1, \Omega}\right),
$$

for $t=0,1$

Proof. Let $\left(\boldsymbol{v}_{1}, q_{0}\right)=\left(\mathcal{C}_{H}(\boldsymbol{u}), \Pi_{H}(p)\right)$; then

$$
\left\|\left(\boldsymbol{u}-\boldsymbol{u}_{1, h}, p-p_{0, h}\right)\right\|_{H} \leq\left\|\left(\boldsymbol{u}-\boldsymbol{v}_{1}, p-q_{0}\right)\right\|_{H}+\left\|\left(\boldsymbol{u}_{1, h}-\boldsymbol{v}_{1}, p_{0, h}-q_{0}\right)\right\|_{H} .
$$

The first term is easily estimated using (3.2), (3.4) and (3.6). For the second one, from Lemma 4.4 there exists $\left(\boldsymbol{w}_{1}, t_{0}\right) \in \mathbf{V}_{H} \times Q_{H}$ satisfying $\left\|\left(\boldsymbol{w}_{1}, t_{0}\right)\right\|_{H}=1$ and

$$
\begin{aligned}
& \beta_{2}\left\|\left(\boldsymbol{u}_{1, h}-\boldsymbol{v}_{1}, p_{0, h}-q_{0}\right)\right\|_{H} \leq \boldsymbol{B}_{h}\left(\left(\boldsymbol{u}_{1, h}-\boldsymbol{v}_{1}, p_{0, h}-q_{0}\right),\left(\boldsymbol{w}_{1}, t_{0}\right)\right) \\
& \quad=\boldsymbol{B}_{h}\left(\left(\boldsymbol{u}_{1, h}-\boldsymbol{u}, p_{0, h}-p\right),\left(\boldsymbol{w}_{1}, t_{0}\right)\right)+\boldsymbol{B}_{h}\left(\left(\boldsymbol{u}-\boldsymbol{v}_{1}, p-q_{0}\right),\left(\boldsymbol{w}_{1}, t_{0}\right)\right) .
\end{aligned}
$$

Now, since $\left(p-q_{0}, \nabla \cdot \boldsymbol{w}_{1}\right)_{\Omega}=0$, it follows that

$$
\begin{aligned}
\boldsymbol{B}_{h}\left(\left(\boldsymbol{u}-\boldsymbol{v}_{1}, p-q_{0}\right),\left(\boldsymbol{w}_{1}, t_{0}\right)\right) \\
=\sum_{K \in \mathcal{T}_{H}} \sigma\left(\nabla p_{h}\left(\boldsymbol{u}-\boldsymbol{v}_{1}\right), \nabla p_{h}\left(\boldsymbol{w}_{1}\right)\right)_{K}-\left(t_{0}, \nabla \cdot\left(\boldsymbol{u}-\boldsymbol{v}_{1}\right)\right)_{\Omega} \\
\quad-\sum_{K \in \mathcal{T}_{H}} \tau_{F}\left(\llbracket p-q_{0} \rrbracket, \llbracket t_{0} \rrbracket\right)_{F} \\
\leq \sum_{K \in \mathcal{T}_{H}} \sigma\left|p_{h}\left(\boldsymbol{u}-\boldsymbol{v}_{1}\right)\right|_{1, K}\left|p_{h}\left(\boldsymbol{w}_{1}\right)\right|_{1, K}+\left\|t_{0}\right\|_{0, \Omega}\left\|\nabla \cdot\left(\boldsymbol{u}-\boldsymbol{v}_{1}\right)\right\|_{0, \Omega} \\
\quad+\sum_{F \in \mathcal{E}_{H}} \tau_{F}\left\|\llbracket p-q_{0} \rrbracket\right\|_{0, F}\left\|\llbracket t_{0} \rrbracket\right\|_{0, F} .
\end{aligned}
$$


Hence, using (4.11), (3.2), (3.4), (3.6) and $\left\|\left(\boldsymbol{w}_{1}, t_{0}\right)\right\|_{H}=1$, we arrive at

$$
\begin{aligned}
\boldsymbol{B}_{h}\left(\left(\boldsymbol{u}-\boldsymbol{v}_{1}, p-q_{0}\right),\left(\boldsymbol{w}_{1}, t_{0}\right)\right) \leq \sum_{K \in \mathcal{T}_{H}} \sigma\left\|\boldsymbol{u}-\boldsymbol{v}_{1}\right\|_{0, K}\left\|\boldsymbol{w}_{1}\right\|_{0, K} \\
+\left\|t_{0}\right\|_{0, \Omega}\left\|\nabla \cdot\left(\boldsymbol{u}-\boldsymbol{v}_{1}\right)\right\|_{0, \Omega}+\sum_{F \in \mathcal{E}_{H}} \tau_{F}\left\|\llbracket p-q_{0} \rrbracket\right\|_{0, F}\left\|\llbracket t_{0} \rrbracket\right\|_{0, F} \\
\quad \leq C\left(\sum_{K \in \mathcal{T}_{H}} \sigma H_{K}^{4}|\boldsymbol{u}|_{2, \omega_{K}}^{2}+\frac{H^{2} \sigma}{\alpha}|\boldsymbol{u}|_{2, \Omega}^{2}+\frac{H^{2}}{\sigma}|p|_{1, \Omega}^{2}\right)^{\frac{1}{2}}
\end{aligned}
$$

To bound the remaining term, we use the fact that $\llbracket p \rrbracket=0$ and the definition of $\boldsymbol{B}_{h}$ and $\boldsymbol{B}$ to obtain

$$
\begin{aligned}
\boldsymbol{B}_{h}\left(\left(\boldsymbol{u}_{1, h}-\boldsymbol{u}, p_{0, h}-p\right),\left(\boldsymbol{w}_{1}, t_{0}\right)\right)=\boldsymbol{B}_{h}\left(\left(\boldsymbol{u}_{1, h}, p_{0, h}\right),\left(\boldsymbol{w}_{1}, t_{0}\right)\right)-\boldsymbol{B}_{h}\left((\boldsymbol{u}, p),\left(\boldsymbol{w}_{1}, t_{0}\right)\right) \\
=\mathbf{F}_{h}\left(\boldsymbol{w}_{1}, t_{0}\right)-\left\{\sum_{K \in \mathcal{T}_{H}} \sigma\left(\nabla p_{h}(\boldsymbol{u}), \nabla p_{h}\left(\boldsymbol{w}_{1}\right)\right)_{K}-\left(p, \nabla \cdot \boldsymbol{w}_{1}\right)_{\Omega}-\left(t_{0}, \nabla \cdot \boldsymbol{u}\right)_{\Omega}\right\} \\
=\mathbf{F}_{h}\left(\boldsymbol{w}_{1}, t_{0}\right)-\left\{\sum_{K \in \mathcal{T}_{H}} \sigma\left(\nabla p_{e}^{M}(\boldsymbol{u}), \nabla p_{e}^{M}\left(\boldsymbol{w}_{1}\right)\right)_{K}-\left(p, \nabla \cdot \boldsymbol{w}_{1}\right)_{\Omega}-\left(t_{0}, \nabla \cdot \boldsymbol{u}\right)_{\Omega}\right\} \\
+\sum_{K \in \mathcal{T}_{H}} \sigma\left[\left(\nabla p_{e}^{M}(\boldsymbol{u}), \nabla p_{e}^{M}\left(\boldsymbol{w}_{1}\right)\right)_{K}-\left(\nabla p_{h}(\boldsymbol{u}), \nabla p_{h}\left(\boldsymbol{w}_{1}\right)\right)_{K}\right] \\
=\mathbf{F}_{h}\left(\boldsymbol{w}_{1}, t_{0}\right)-\boldsymbol{B}\left((\boldsymbol{u}, p),\left(\boldsymbol{w}_{1}, t_{0}\right)\right) \\
+\sum_{K \in \mathcal{T}_{H}} \sigma\left[\left(\nabla p_{e}^{M}(\boldsymbol{u})-\nabla p_{h}(\boldsymbol{u}), \nabla p_{e}^{M}\left(\boldsymbol{w}_{1}\right)\right)_{K}+\left(\nabla p_{h}(\boldsymbol{u}), \nabla p_{e}^{M}\left(\boldsymbol{w}_{1}\right)-\nabla p_{h}\left(\boldsymbol{w}_{1}\right)\right)_{K}\right] .
\end{aligned}
$$

Next, using the Galerkin orthogonality in each element $K$ we get $\left(\nabla p_{h}(\boldsymbol{u}), \nabla p_{e}^{M}\left(\boldsymbol{w}_{1}\right)\right.$ $\left.-\nabla p_{h}\left(\boldsymbol{w}_{1}\right)\right)_{K}=0$, and then from Lemmas 3.6 and 4.1 we arrive at

$$
\begin{aligned}
\boldsymbol{B}_{h}\left(\left(\boldsymbol{u}_{1, h}-\boldsymbol{u}, p_{0, h}-p\right),\left(\boldsymbol{w}_{1}, t_{0}\right)\right)=\mathbf{F}_{h}\left(\boldsymbol{w}_{1}, t_{0}\right)-\mathbf{F}\left(\boldsymbol{w}_{1}, t_{0}\right) & \\
& -\boldsymbol{B}\left(\left(\boldsymbol{u}-\boldsymbol{u}_{1}, p-p_{0}\right),\left(\boldsymbol{w}_{1}, t_{0}\right)\right)+\sum_{K \in \mathcal{T}_{H}} \sigma\left(\nabla p_{e}^{M}(\boldsymbol{u})-\nabla p_{h}(\boldsymbol{u}), \nabla p_{e}^{M}\left(\boldsymbol{w}_{1}\right)\right)_{K} \\
= & \sum_{K \in \mathcal{T}_{H}}\left[\left(\nabla p_{e}^{g}-\nabla p_{h}^{g}, \boldsymbol{w}_{1}\right)_{K}+\sigma\left(\nabla p_{e}^{M}(\boldsymbol{u})-\nabla p_{h}(\boldsymbol{u}), \nabla p_{e}^{M}\left(\boldsymbol{w}_{1}\right)\right)_{K}\right] \\
& -\boldsymbol{B}\left(\left(\boldsymbol{u}-\boldsymbol{u}_{1}, p-p_{0}\right),\left(\boldsymbol{w}_{1}, t_{0}\right)\right) \\
\leq & \sum_{K \in \mathcal{T}_{H}}\left[\left|p_{e}^{g}-p_{h}^{g}\right|_{1, K}\left\|\boldsymbol{w}_{1}\right\|_{0, K}+\sigma\left|p_{e}^{M}(\boldsymbol{u})-p_{h}(\boldsymbol{u})\right|_{1, K}\left|p_{e}^{M}\left(\boldsymbol{w}_{1}\right)\right|_{1, K}\right] \\
& +C \sigma H|\boldsymbol{u}|_{1, \Omega}\left\|\boldsymbol{w}_{1}\right\|_{0, \Omega} \\
\leq & \sum_{K \in \mathcal{T}_{H}} C\left[\sigma^{-1} h\left|p_{e}^{g}\right|_{2, K}\left\|\boldsymbol{w}_{1}\right\|_{0, K}+\sigma h\left|p_{e}^{M}(\boldsymbol{u})\right|_{2, K}\left\|\boldsymbol{w}_{1}\right\|_{0, K}\right]+C \sigma H|\boldsymbol{u}|_{1, \Omega}\left\|\boldsymbol{w}_{1}\right\|_{0, \Omega} \\
\leq & C\left(\frac{h H^{t}}{\sqrt{\sigma}}|g|_{t, \Omega}+\sqrt{\sigma}(H+h)|\boldsymbol{u}|_{1, \Omega}\right)
\end{aligned}
$$

and the result follows. 

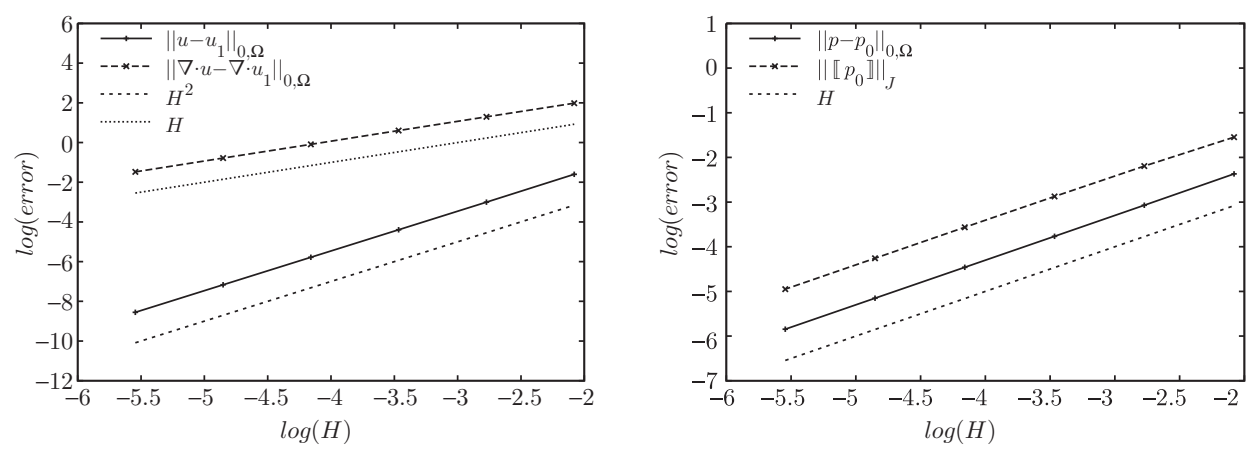

Figure 2. Example I: Convergence history for the velocity field and its divergence (left) and for the pressure and jump (right).

Remark 4.6. We finally remark that Lemma 2.7 is also valid for the solution of the fully discrete problem (4.8); i.e., the following holds:

$$
\int_{K} \nabla \cdot\left(\boldsymbol{u}_{1, h}+\boldsymbol{u}_{e}^{D}\right)-g=0 \quad \forall K \in \mathcal{T}_{H},
$$

where, here $\boldsymbol{u}_{e}^{D}=\mathcal{D}_{K}^{\boldsymbol{u}}\left(\llbracket p_{0, h} \rrbracket\right)$.

\section{Numerical EXPERIMENTS}

We are interested in the numerical validation of the fully discrete method (4.8). The validations are performed through three series of numerical tests. The first two experiments aim to compare the solution provided by (4.8) with available analytical solutions. For both tests the local mass conservation feature is verified and an analysis of sensitivity with respect to $\alpha_{F}$ is performed. The robustness of the method to face problems out of the scope is assessed in the final test by solving the so-called five-spot benchmark. Finally, and just to simplify the notation, the solution $\left(\boldsymbol{u}_{1, h}, p_{0, h}\right)$ of (4.8) will be denoted simply by $\left(\boldsymbol{u}_{1}, p_{0}\right)$.

5.1. Analytical solution: First study. The domain is $\Omega=(0,1) \times(0,1)$ for the first test and for all remaining tests as well. Moreover, we set $\sigma=1$ and set the exact pressure equal to $p(x, y)=\cos (2 \pi x) \cos (2 \pi y), \boldsymbol{u}=-\nabla p$, and thus $b=0$ and $g=\nabla \cdot \boldsymbol{u}=8 \pi^{2} \cos (2 \pi x) \cos (2 \pi y)$. In Figure 2 we report the errors on velocity and pressure in a sequence of structured meshes using $\alpha_{F}=0.1$, and we observe optimal convergence of all quantities as $H \rightarrow 0$ in their respective natural norms, which is in accordance with the theoretical results. For all the examples, we use the notation

$$
\left\|\llbracket p_{0} \rrbracket\right\|_{J}=\left\{\sum_{F \in \mathcal{E}_{H}} H_{F}\left\|\llbracket p_{0} \rrbracket\right\|_{0, F}^{2}\right\}^{\frac{1}{2}} .
$$

Furthermore, in Table1 1 we study the local mass conservation feature for $\boldsymbol{u}_{1}+\boldsymbol{u}_{e}^{D}$. For that we define the quantity

$$
M_{e}:=\max _{K \in \mathcal{T}_{H}} \frac{\left|\int_{K}\left(\nabla \cdot\left(\boldsymbol{u}_{1}+\boldsymbol{u}_{e}^{D}\right)-g\right) d x\right|}{|K|}
$$


and observe that we recover the local mass conservation property updating the linear velocity field by the multiscale velocity $\boldsymbol{u}_{e}^{D}$.

TABLE 1. Example I: Relative local mass conservation error.

\begin{tabular}{cccccc}
\hline$H$ & $6.25 \times 10^{-2}$ & $3.12 \times 10^{-2}$ & $1.56 \times 10^{-2}$ & $7.8 \times 10^{-3}$ & $3.9 \times 10^{-3}$ \\
$M_{e}$ & $1.4 \times 10^{-12}$ & $1.3 \times 10^{-12}$ & $3.6 \times 10^{-12}$ & $6.9 \times 10^{-12}$ & $7 \times 10^{-11}$ \\
\hline
\end{tabular}

Next, a study of sensitivity of the numerical error with respect to $\alpha_{F}$ is performed in Table 2 for a fixed mesh, where we observe that the errors remain independent of the parameter as long as $\alpha_{F}$ stays of order 1. That agrees with the assumption that $\tau_{F}$ must be of order $H_{F}$, as predicted by the theory. We also perform a convergence study for all the variables using different values for $\alpha_{F}$. The results are depicted in Figures 3 and 4 , where we can see that the errors are practically unaffected by the value of $\alpha_{F}$.

TABLE 2. Example I: The sensitivity of the errors with respect to $\alpha_{F}$.

\begin{tabular}{lccccc}
$\alpha_{F}$ & $\left\|\left(\boldsymbol{u}-\boldsymbol{u}_{1}, p-p_{0}\right)\right\|_{H}$ & $\left\|\boldsymbol{u}-\boldsymbol{u}_{1}\right\|_{0, \Omega}$ & $\left\|\nabla \cdot \boldsymbol{u}-\nabla \cdot \boldsymbol{u}_{1}\right\|_{0, \Omega}$ & $\left\|p-p_{0}\right\|_{0, \Omega}$ & $\left\|\llbracket p_{0} \rrbracket\right\|_{J}$ \\
\hline $10^{-6}$ & 1.8380 & 0.012 & 1.826 & 0.023 & 0.056 \\
$10^{-4}$ & 1.8380 & 0.012 & 1.826 & 0.023 & 0.056 \\
$10^{-2}$ & 1.8380 & 0.012 & 1.826 & 0.023 & 0.056 \\
0.1 & 1.8459 & 0.012 & 1.826 & 0.023 & 0.056 \\
1 & 1.9220 & 0.013 & 1.830 & 0.023 & 0.056 \\
\hline
\end{tabular}

Our next objective is to perform a comparison of the performance of (4.8) with the lowest order Raviart-Thomas mixed finite element method $R T_{0} / Q_{H}$ (cf. [26, 11]). The comparison is performed in Figures 5 and 6 . We can see from the results that clearly (4.8) is far more accurate than the Raviart-Thomas method in the $L^{2}(\Omega)^{2}$ norm of the velocity field, and, thanks to the mass conservation property for $\boldsymbol{u}_{1}+\boldsymbol{u}_{e}^{D}$, when the velocity field is updated with $\boldsymbol{u}_{e}^{D}$, the errors in the divergence are identical. Both methods seem to perform equally good regarding the errors in the pressure field. We want to stress the fact that the solution of (4.8) involves fewer degrees of freedom than $R T_{0} / Q_{H}$ when the same mesh is used.
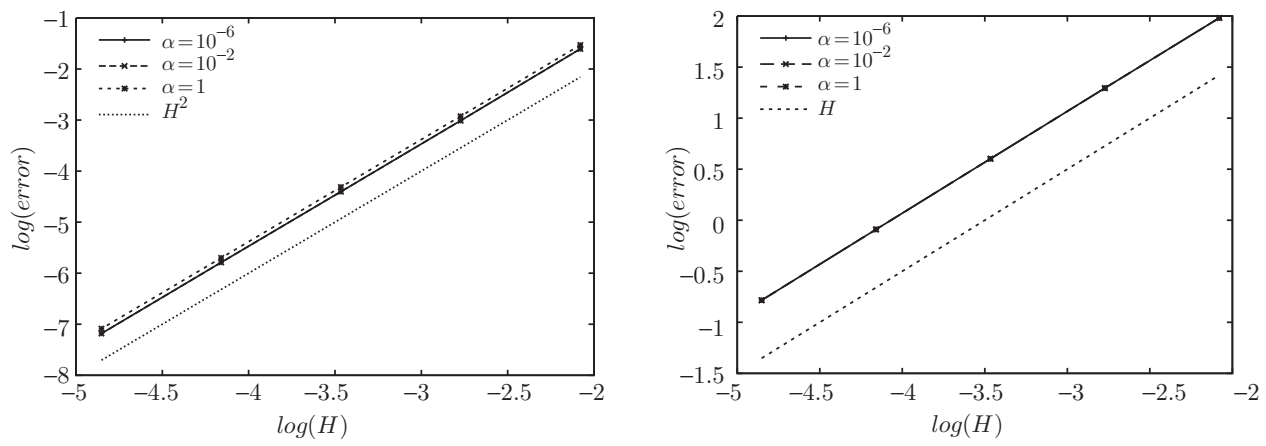

Figure 3. Example I: Convergence history for the velocity field (left) and its divergence (right) for different values of $\alpha_{F}$. 

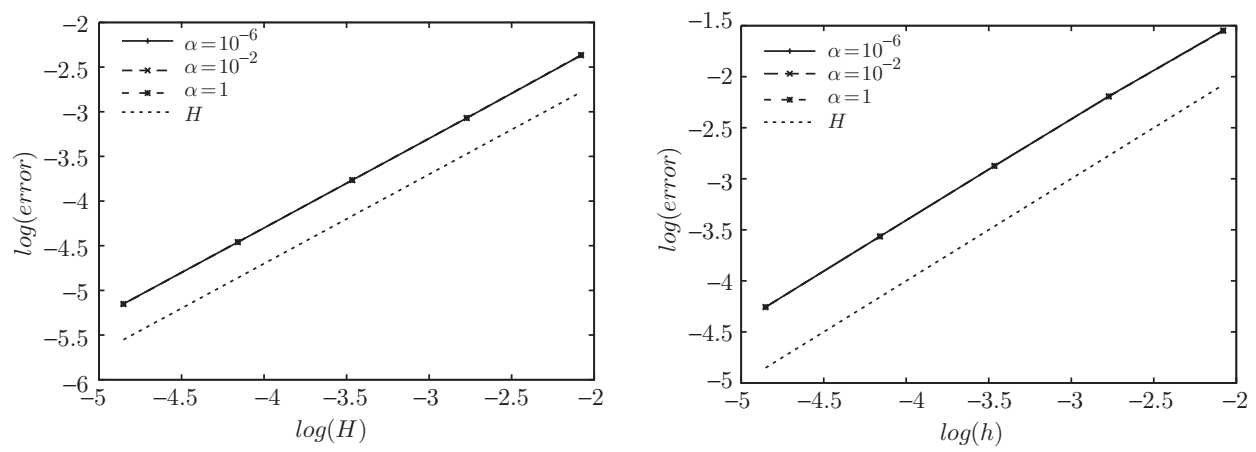

Figure 4. Example I: Convergence history for the pressure (left) and jump (right) for different values of $\alpha_{F}$.
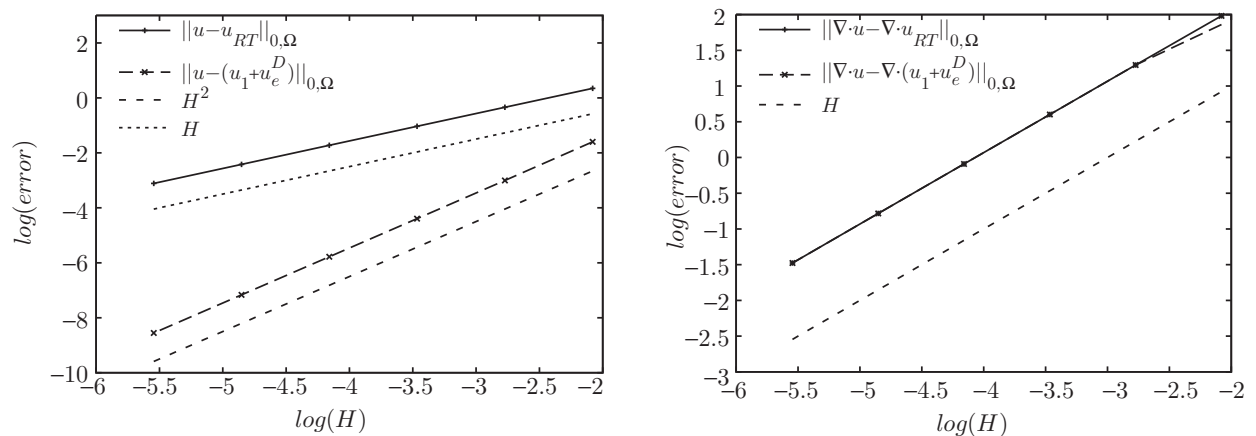

Figure 5. Example I: Convergence history for the velocity (left) and its divergence (right) for (4.8) and $R T_{0} / Q_{H}$.

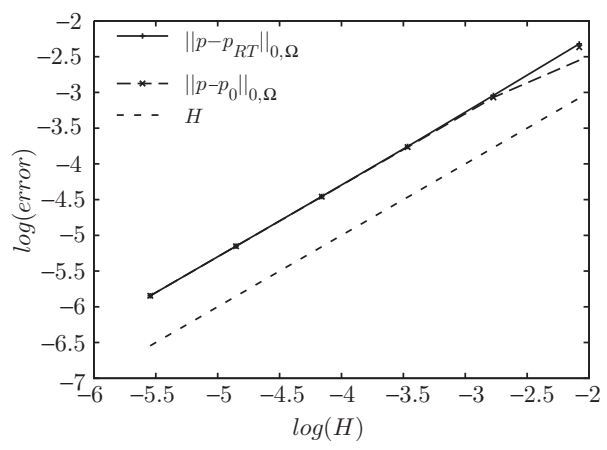

Figure 6. Example I: Convergence history for the pressure for (4.8) and $R T_{0} / Q_{H}$. 


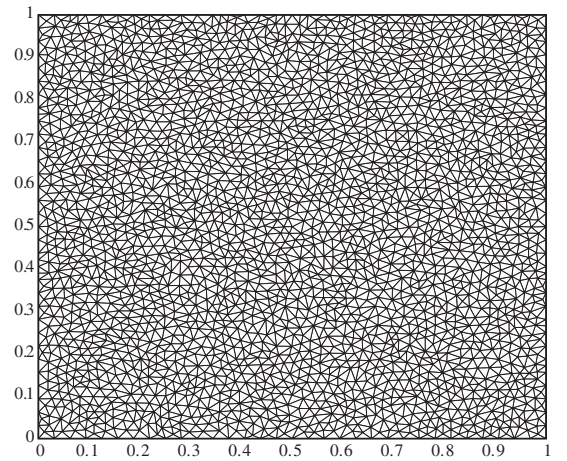

Figure 7 . The unstructured mesh.

Finally, the unstructured mesh of Figure 7 containing approximately 5000 elements, is adopted. The sensitivity of error in terms of parameter $\alpha_{F}$ presents a different behavior than before. Nevertheless, since there is a loss of stability when $\alpha_{F}$ is small, the unexpected robust error behavior when structured meshes are used is no longer preserved. The results are reported in Table 3, where we observe that the individual norms are not independent of $\alpha_{F}$, but the whole $\|\cdot\|_{H}$ norm of the error seems robust with respect to $\alpha_{F}$.

TABLE 3. Example I: The sensitivity of the errors with respect to $\alpha_{F}$ using an unstructured mesh.

\begin{tabular}{lccccc}
$\alpha_{F}$ & $\left\|\boldsymbol{u}-\boldsymbol{u}_{1}\right\|_{0, \Omega}$ & $\left\|\nabla \cdot \boldsymbol{u}-\nabla \cdot \boldsymbol{u}_{1}\right\|_{0, \Omega}$ & $\left\|p-p_{0}\right\|_{0, \Omega}$ & $\left\|\llbracket p_{0} \rrbracket\right\|_{J}$ & $\left\|\left(\boldsymbol{u}-\boldsymbol{u}_{1}, p-p_{0}\right)\right\|_{H}$ \\
\hline $10^{-3}$ & 0.6099 & 1.6741 & 8.0277 & 17.6551 & 1.8843 \\
$10^{-2}$ & 0.3017 & 1.6771 & 1.8341 & 2.6921 & 1.7349 \\
0.1 & 0.1389 & 1.6840 & 0.2585 & 0.4186 & 1.6968 \\
1 & 0.0654 & 1.7011 & 0.0373 & 0.0821 & 1.7047 \\
10 & 0.0366 & 1.8013 & 0.0228 & 0.0541 & 1.8112 \\
\hline
\end{tabular}

5.2. Second analytical solution: A divergence-free velocity field. For the following example we consider a divergence-free velocity field. More precisely, the problem is set up as in the first test. The exact pressure is now $p(x, y)=x-x^{2}-1 / 6$ and the velocity field is

$$
\boldsymbol{u}=\left(x^{2}(1-x)^{2}\left(2 y-6 y^{2}+4 y^{3}\right),-y^{2}(1-y)^{2}\left(2 x-6 x^{2}+4 y^{3}\right)\right)^{t} .
$$

The source term is then

$$
\boldsymbol{f}=\left(x^{2}(1-x)^{2}\left(2 y-6 y^{2}+4 y^{3}\right)+1-2 x,-y^{2}(1-y)^{2}\left(2 x-6 x^{2}+4 y^{3}\right)\right)^{t},
$$

and the boundary condition is $b=0$. Since the source term $f$ is no longer a constant function in $K$, we must consider the enhancement of $\mathcal{M}_{K}^{u}(\boldsymbol{f})$, i.e., we must add the term $\sum_{K \in \mathcal{T}_{H}}\left(\mathcal{M}_{K}^{u}(-\boldsymbol{f}), \sigma \boldsymbol{v}_{1}\right)_{K}$ to the right hand side. From the definition of the 

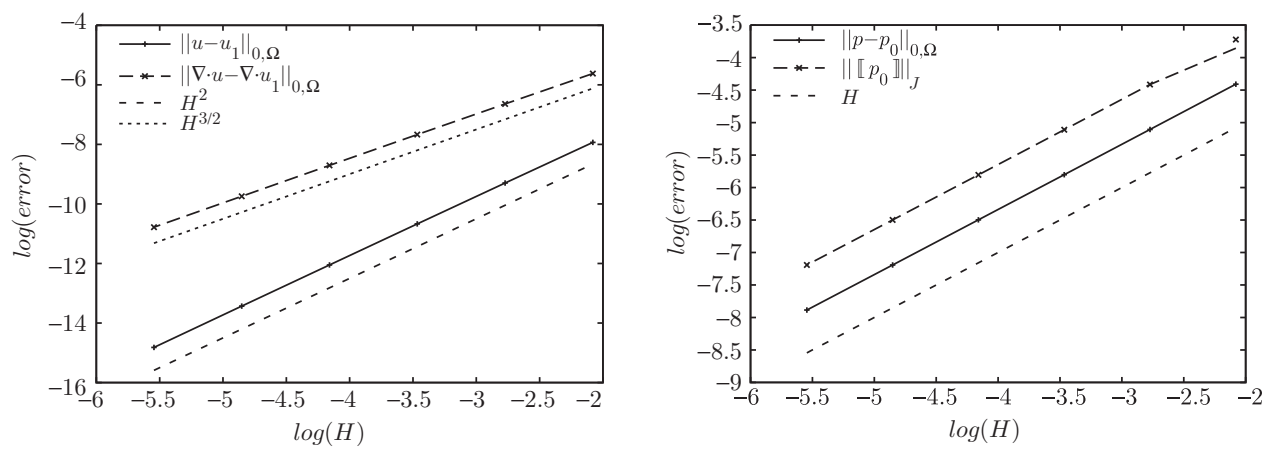

FiguRE 8. Example II: Convergence history for the velocity field and its divergence (left) and for the pressure and jump (right).

operator $\mathcal{M}_{K}^{u}$ we rewrite the right hand side of the equation as

$$
\begin{aligned}
\left(\boldsymbol{f}, \boldsymbol{v}_{1}\right)_{\Omega}+\sum_{K \in \mathcal{T}_{H}}\left(\sigma \boldsymbol{u}_{e}^{M}(-\boldsymbol{f}), \boldsymbol{v}_{1}\right)_{K} & =\left(\boldsymbol{f}, \boldsymbol{v}_{1}\right)_{\Omega}+\sum_{K \in \mathcal{T}_{H}}\left(-\boldsymbol{f}-\nabla p_{e}^{M}(-\boldsymbol{f}), \boldsymbol{v}_{1}\right)_{K} \\
& =\sum_{K \in \mathcal{T}_{H}}\left(\nabla p_{e}^{M}(\boldsymbol{f}), \boldsymbol{v}_{1}\right)_{K}
\end{aligned}
$$

where $p_{e}^{M}(\boldsymbol{f})$ is solution of the local problem

$$
-\Delta p_{e}^{M}=-\nabla \cdot \boldsymbol{f} \quad \text { in } \quad K, \quad \partial_{\boldsymbol{n}} p_{e}^{M}=\boldsymbol{f} \cdot \boldsymbol{n} \quad \text { on } \quad \partial K .
$$

Now, considering the local problems and the conservation mass property, we have $\nabla \cdot \boldsymbol{u}_{1}+\nabla \cdot \boldsymbol{u}_{e}^{D}=0$ at the element level, leading to

$$
\left.\nabla \cdot \boldsymbol{u}_{1}\right|_{K}=-\frac{1}{|K|} \sum_{F \in \partial K} \frac{\alpha_{F} H_{F}}{\sigma} \int_{F} \llbracket p_{0} \rrbracket \boldsymbol{n} \cdot \boldsymbol{n}_{F} \cdot
$$

Hence, we do not expect in general the error for the divergence of the velocity field to have a good behavior with respect to the parameter $\alpha_{F}$, and we expect a small variation in the norm $H$ since the divergence of the velocity field becomes more important as the parameter $\alpha_{F}$ is of order one (see Table 5 and Figures 9 and 10).

The results concerning the errors on velocity and pressure are depicted in Figure 8 using $\alpha_{F}=0.1$. In this figure we observe an $H^{3 / 2}$ convergence for the velocity field in the $H(d i v, \Omega)$ norm, which is higher than the expected rate of convergence given by the analysis. This is a good thing when compared to the Raviart-Thomas method, in which the discrete velocity field is exactly divergence-free. Of course, when updated with the enrichment function $\boldsymbol{u}_{e}^{D}$, the velocity field becomes exactly divergence-free (see Table 4 for the mass-conservation results). The sensitivity of the error with respect to $\alpha_{F}$ is performed in Table 5 , and, as before, we study the convergence of the method for different choices of $\alpha_{F}$ and we report the results in Figures 9 and 10, where we observe that the errors in divergence are affected by the value of $\alpha_{F}$, while the rest seem fairly independent of $\alpha_{F}$. 
TABLE 4. Example II: Relative local mass conservation error.

\begin{tabular}{cccccc}
\hline$H$ & $6.25 \times 10^{-2}$ & $3.12 \times 10^{-2}$ & $1.56 \times 10^{-2}$ & $7.8 \times 10^{-3}$ & $3.9 \times 10^{-3}$ \\
$M_{e}$ & $1.04 \times 10^{-15}$ & $1.03 \times 10^{-15}$ & $9.11 \times 10^{-15}$ & $1.41 \times 10^{-14}$ & $6.3 \times 10^{-15}$ \\
\hline
\end{tabular}

TABLE 5. Example II: The sensitivity of errors with respect to $\alpha_{F}$.

\begin{tabular}{lccccc}
$\alpha_{F}$ & $\left\|\left(\boldsymbol{u}-\boldsymbol{u}_{1}, p-p_{0}\right)\right\|_{H}$ & $\left\|\boldsymbol{u}-\boldsymbol{u}_{1}\right\|_{0, \Omega}$ & $\left\|\nabla \cdot \boldsymbol{u}-\nabla \cdot \boldsymbol{u}_{1}\right\|_{0, \Omega}$ & $\left\|p-p_{0}\right\|_{0, \Omega}$ & $\left\|\llbracket p_{0} \rrbracket\right\|_{J}$ \\
\hline $10^{-6}$ & 0.0090 & $5.7 \times 10^{-5}$ & $4.6 \times 10^{-9}$ & 0.003 & 0.006 \\
$10^{-4}$ & 0.0090 & $5.7 \times 10^{-5}$ & $4.6 \times 10^{-7}$ & 0.003 & 0.006 \\
$10^{-2}$ & 0.0090 & $5.7 \times 10^{-5}$ & $4.6 \times 10^{-5}$ & 0.003 & 0.006 \\
0.1 & 0.0094 & $6 \times 10^{-5}$ & $4.6 \times 10^{-4}$ & 0.003 & 0.006 \\
1 & 0.0013 & $1.9 \times 10^{-4}$ & $4.6 \times 10^{-3}$ & 0.003 & 0.006 \\
\hline
\end{tabular}
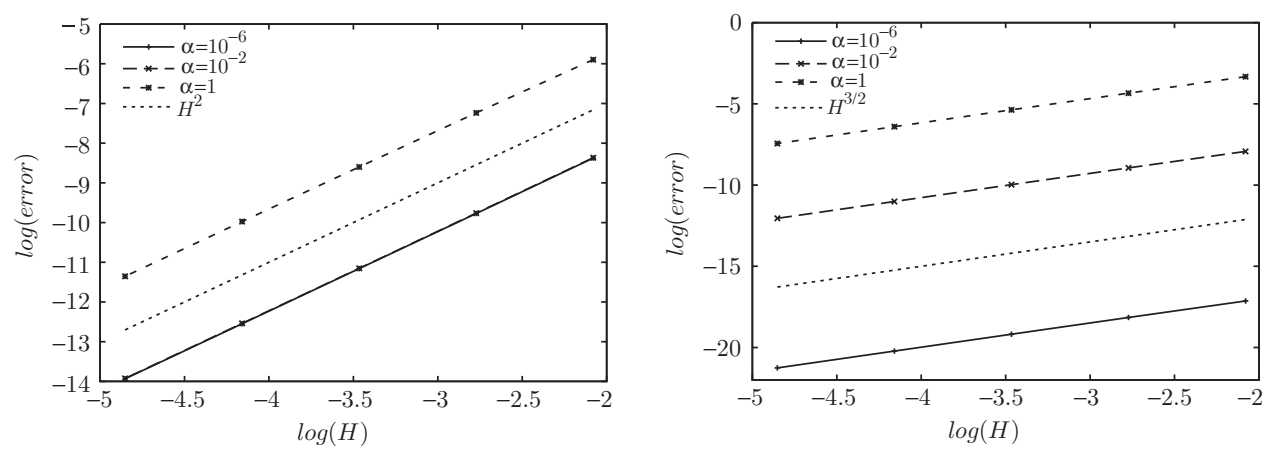

Figure 9. Example II: Convergence history for the velocity field (left) and its divergence (right) for different values of $\alpha_{F}$.
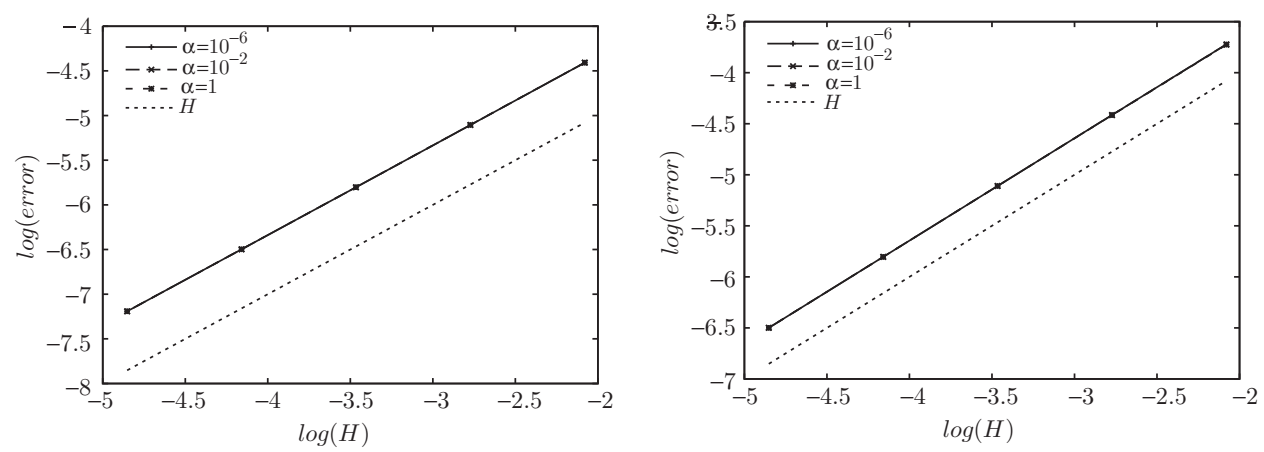

Figure 10. Example II: Convergence history for the pressure (left) and jump (right) for different values of $\alpha_{F}$. 

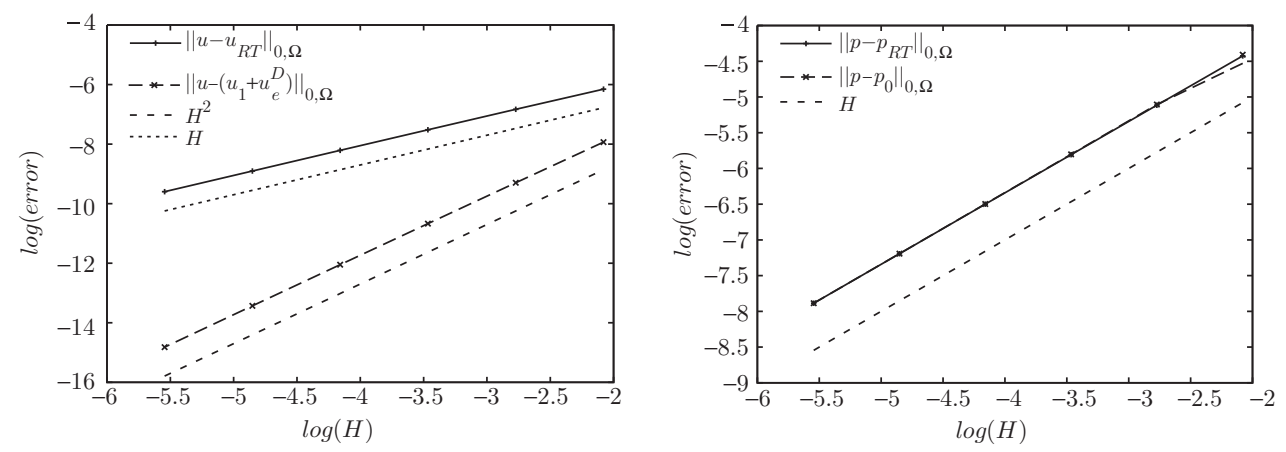

Figure 11. Example II: Convergence history for the velocity (left) and for the pressure (right) for (4.8) and $R T_{0} / Q_{H}^{0}$.

Now we perform a comparison of (4.8) with the lowest order Raviart-Thomas mixed method $R T_{0} / Q_{H}$, where we get better precision for the velocity field, as before, and the errors for the pressure seem very close as well. The results are depicted in Figure 11.

5.3. The five-spot problem. Due to its practical importance in oil recovery, the quarter five-spot problem has served as a paradigm to validate stability and accuracy of numerical methods for the Darcy model. This problem is now addressed by considering zero source term $\boldsymbol{f}$ and $\sigma=1$ in a unit square domain, and instead of modeling injection and production of a non-zero source term $g$, we consider a nonhomogeneous boundary condition for the velocity such that its normal component is equal to $\frac{1}{4 H_{F}}$ at points $(0,0)$ and $(1,1)$. This delta of Dirac is linearly approached on the edges sharing such points. The solution obtained is depicted in Figures 12 15. where we observe the total absence of oscillations in the solution. The constant $\alpha_{F}$ is again fixed equal to 0.1 . In Table 6 we study the local mass conservation feature regarding the enhanced method.

TABLE 6. Five-spot problem: Relative local mass conservation error.

\begin{tabular}{rccccc}
\hline$h$ & $6.25 \times 10^{-2}$ & $3.12 \times 10^{-2}$ & $1.56 \times 10^{-2}$ & $7.8 \times 10^{-3}$ & $3.9 \times 10^{-3}$ \\
$M_{e} 4.11 \times 10^{-12}$ & $2.3 \times 10^{-12}$ & $7.29 \times 10^{-12}$ & $1.34 \times 10^{-11}$ & $1.32 \times 10^{-9}$ \\
\hline
\end{tabular}




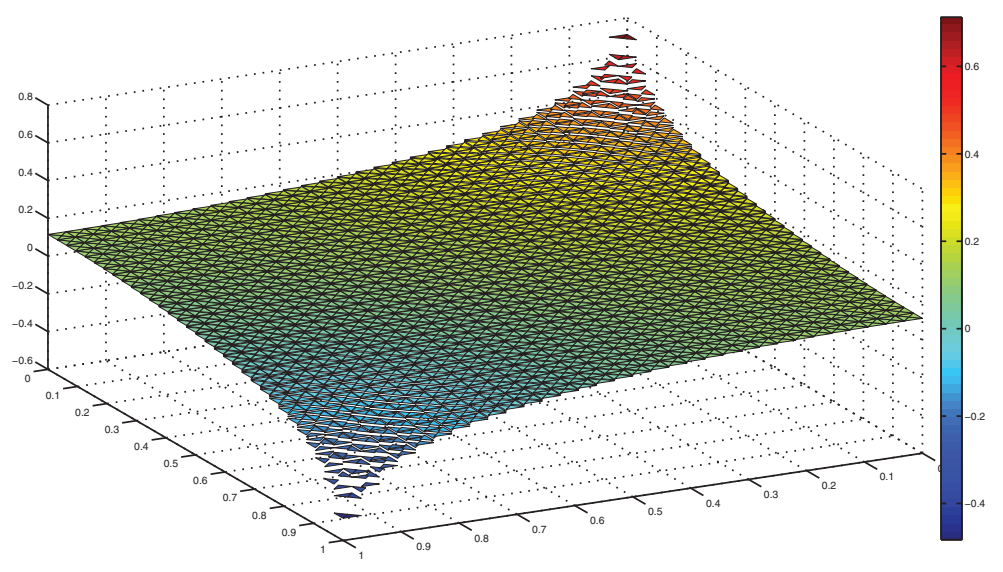

Figure 12. Five-spot problem: Profile of pressure.

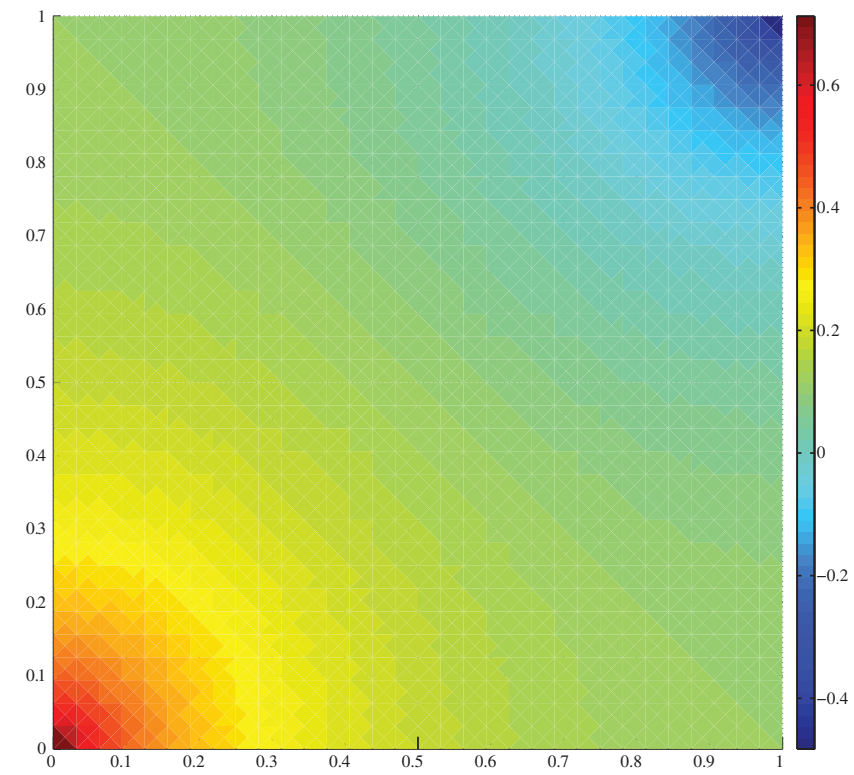

Figure 13. Five-spot problem: Isovalues of the pressure. 


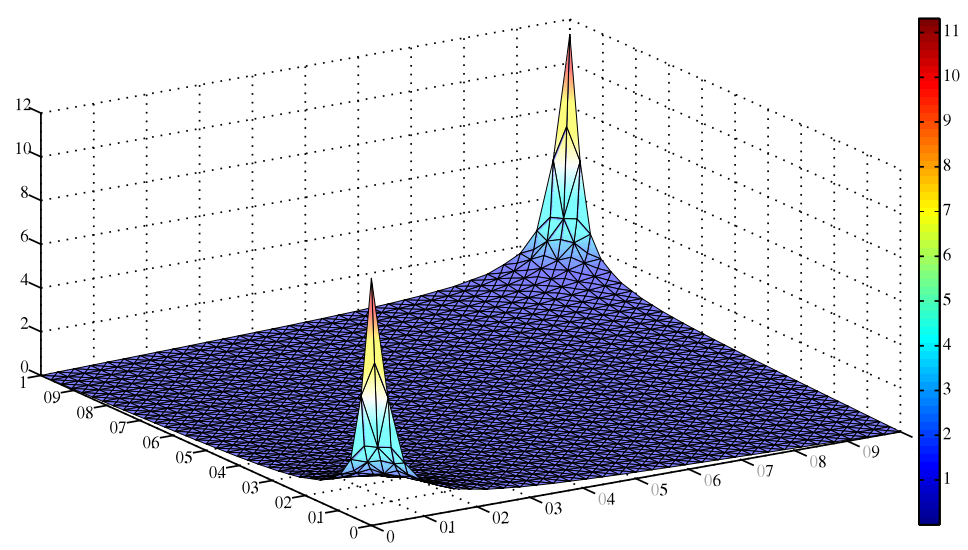

Figure 14. Five-spot problem: The 2-norm of the velocity.
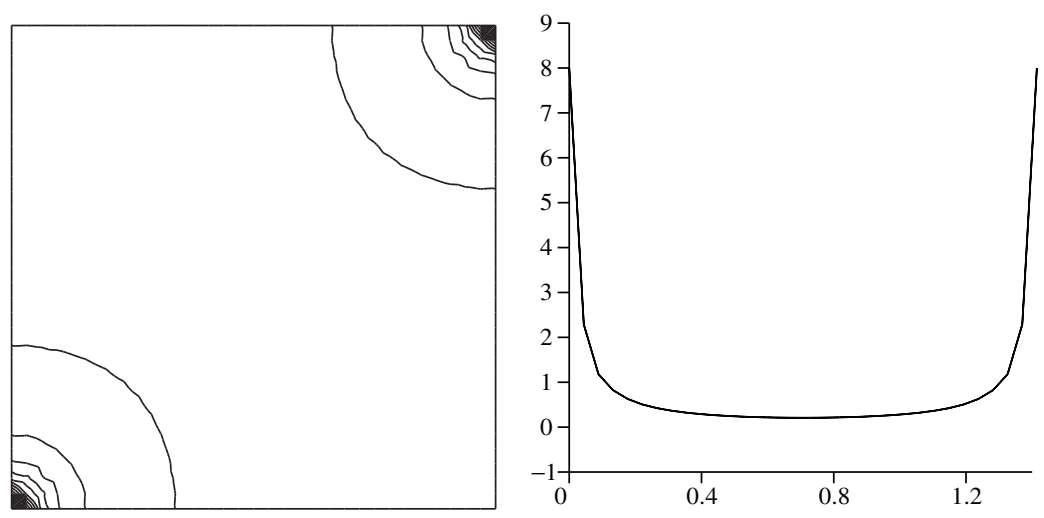

Figure 15. Five-spot problem: Isovalues for $\left|\boldsymbol{u}_{1}\right|$ and a crosssection along the line $y=x$.

\section{Conclusion}

To adopt the original operator along with boundary conditions built on imposing continuity for the pressure and the flux appears to be the correct form to model unsolved scales in terms of resolved ones, which means, to recover stability and optimality through the Petrov-Galerkin augmenting space approach. In addition, by incorporating such unsolved scales into the finite element method all the desired features of the original spaces, such as local mass conservation and nodal values for the velocity, are still preserved. When it comes to solving the local problems, the proposed method compromises accuracy with low computational cost. Thus, our method appears as a competitive alternative for tackling more complex flows where analytical solutions are out of reach, such as oscillating coefficients. Higher order pairs of interpolation spaces will also demand the two-level approach, but in those cases, the boundary condition for the local problems ought to include further control on the gradient of the pressure. Finally, enriched methods seem to show an 
intrinsic relationship with some discontinuous finite element methods. This subject will be addressed in forthcoming works.

\section{ACKNOWLEDGMEnTs}

This work was partially carried out during the stay of Frédéric Valentin at the Center for Computational Mathematics of the University of Colorado at Denver, USA, supported by the NSF/USA Grant No. 0610039. The authors want to thank Leopoldo Franca, Rodolfo Rodríguez and Francisco Javier Sayas for many helpful discussions and comments.

\section{REFERENCES}

1. R. Araya, G. R. Barrenechea, L. P. Franca, and F. Valentin, Stabilization arising from PGEM: A review and further developments, Applied Numerical Mathematics 59 (2009), no. 9, 20652081. MR2532854

2. R. Araya, G. R. Barrenechea, and F. Valentin, Stabilized finite element methods based on multiscale enrichment for the Stokes problem, SIAM J. Numer. Anal. 44 (2006), no. 1, 322348. MR2217385 (2007f:65049)

3. T. Arbogast, Analysis of a two-scale locally conservative subgrid upscaling for elliptic problems, SIAM J. Numer. Anal. 42 (2004), no. 2, 576-598. MR2084227 (2005h:65205)

4. C. Baiocchi, F. Brezzi, and L. P. Franca, Virtual bubbles and Galerkin-Least-Squares type methods (Ga.L.S.), Comput. Methods Appl. Mech. Engrg. 105 (1993), no. 1, 125-141. MR $94 \mathrm{~g}: 65058$

5. G. R. Barrenechea, L. P. Franca, and F. Valentin, A Petrov-Galerkin enriched method: A mass conservative finite element method for the Darcy equation, Computer Methods in Applied Mechanics and Engineering 196 (2007), no. 21-24, 2449-2464. MR2319050 (2008e:76108)

6. _ A symmetric nodal conservative finite element method for the Darcy equation, SIAM J. Numer. Anal. 47 (2009), no. 5, 3652-3677. MR2576515

7. G. R. Barrenechea and F. Valentin, An unusual stabilized finite element method for a generalized Stokes problem, Numer. Math. 92 (2002), no. 4, 653-677. MR2003i:76063

8. _ Relationship between multiscale enrichment and stabilized finite element methods for the generalized Stokes problem, CRAS 341 (2005), no. 10, 635-640. MR2179805

9. P. B. Bochev and M. D. Gunzburger, A locally conservative least-squares method for Darcy flows, Comm. Numer. Methods Engrg. 24 (2008), no. 2, 97-110. MR2369638

10. F. Brezzi, B. Cockburn, L. D. Marini, and E. Süli, Stabilization mechanisms in discontinuous Galerkin finite element methods, Comput. Methods Appl. Mech. Engrg. 195 (2006), no. 25-28, 3293-3310. MR2220920 (2006m:65256)

11. F. Brezzi and M. Fortin, Mixed and hybrid finite element methods, Springer Series in Computational Mathematics, vol. 15, Springer-Verlag, Berlin, New-York, 1991. MR.1115205 (92d:65187)

12. F. Brezzi, L. P. Franca, and A. Russo, Further considerations on residual-free bubbles for advective-diffusive equations, Comput. Methods Appl. Mech. Engrg. 166 (1998), no. 1-2, 2533. MR $99 \mathrm{j}: 65197$

13. F. Brezzi, L. P. Franca, T. J. R. Hughes, and A. Russo, Stabilization techniques and subgrid scale capturing, Proceedings of the Conference "The State of the Art in Numerical Analysis", York, 1-4 April, 1996 (I. Duff, ed.), IMA Conference, Oxford University Press, 1997. MR.1628354 (99f:65162)

14. F. Brezzi and A. Russo, Choosing bubbles for advection-diffusion problems, Math. Models Methods Appl. Sci. 4 (1994), no. 4, 571-587. MR.1291139 (95h:76079)

15. E. Burman and P. Hansbo, A unified stabilized method for Stokes' and Darcy's equations, J. Comput. Appl. Math. 198 (2007), no. 1, 35-51. MR2250387(2007i:65076)

16. Z. Chen and T. Hou, A mixed multiscale finite element method for elliptic problems with oscillating coefficients, Mathematics of Computation 72 (2003), no. 242, 541-576. MR 1954956 (2004a:65147)

17. P. Clément, Approximation by finite element functions using local regularization, RAIRO Anal. Numér. (1975), no. 9, 77-84. MR0400739 (53:4569) 
18. W. E and B. Engquist, The heterogeneous multiscale methods, Commun. Math. Sci. 1 (2003), no. 1, 87-132. MR.2004b:35019

19. A. Ern and J.-L. Guermond, Theory and practice of finite elements, Springer-Verlag, 2004. MR 2050138 (2005d:65002)

20. L. P. Franca, A. L. Madureira, L. Tobiska, and F. Valentin, Convergence analysis of a multiscale finite element method for singularly perturbed problems, SIAM Multiscale Model. and Simul. 4 (2005), no. 3, 839-866. MR.2203943 (2006k:65316)

21. L. P. Franca, A. L. Madureira, and F. Valentin, Towards multiscale functions: Enriching finite element spaces with local but not bubble-like functions, Comput. Methods Appl. Mech. Engrg. 194 (2005), 3006-3021. MR.2142535 (2006a:65159)

22. L. P. Franca, A. Nesliturk, and M. Stynes, On the stability of residual-free bubbles for convection-diffusion problems and their approximation by a two-level finite element method, Comput. Methods Appl. Mech. Engrg. 166 (1998), 35-49. MR1660133 (99k:65107)

23. V. Girault and P. A. Raviart, Finite element methods for Navier-Stokes equations: Theory and algorithms, Springer Series in Computational Mathematics, vol. 5, Springer-Verlag, Berlin, New-York, 1986. MR851383 (88b:65129)

24. T. J. R. Hughes, G. R. Feijoo, L. Mazzei, and J. Quincy, The variational multiscale method - a paradigm for computational mechanics, Computer Methods in Applied Mechanics and Engineering 166 (1998), no. 1-2, 3-24. MR1660141 (99m:65239)

25. L. E. Payne and H. F. Weinberger, An optimal Poincaré inequality for convex domains, Arch. Rational Mech. Anal. 5 (1960), 286-292. MR0117419 (22:8198)

26. P. A. Raviart and J. M. Thomas, A mixed finite element method for 2nd order elliptic problems, Mathematical aspect of finite element methods, no. 606 in Lecture Notes in Mathematics, pp. 292-315, Springer-Verlag, New York, 1977. MR0483555 (58:3547)

27. A. Russo, Residual free bubbles and stabilized methods, Proceedings of the Ninth International Conference on Finite Elements in Fluids - New Trends and Applications (Venice, Italy) (M. Morandi Cecchi, K. Morgan, J. Periaux, B. A. Schrefler, and O. C. Zienkiewicz, eds.), October 1995, pp. 1607-1615.

Department of Mathematics and Statistics, University of Strathclyde, 26 Richmond Street, Glasgow G1 1XH, United Kingdom

E-mail address: alejandro.allendes-flores@strath.ac.uk

Department of Mathematics and Statistics, University of Strathclyde, 26 Richmond Street, Glasgow G1 1XH, United Kingdom

E-mail address: gabriel.barrenechea@strath.ac.uk

Departamento de Matemática, Universidad Técnica Federico Santa María, Casilla 110-V, Valparaíso, Chile

E-mail address: erwin.hernandez@usm.cl

Departamento de Matemática Aplicada e Computacional, laboratório Nacional de Computação Científica, Av. Getúlio Vargas, 333, 25651-070 Petrópolis - RJ, Brazil

E-mail address: valentin@lncc.br 